\section{Arrkivoc

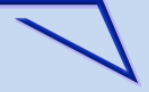

Archive for

Organic Chemistry
The Free Internet Journal

for Organic Chemistry
Paper

Arkivoc 2017, part v, 43-57

\title{
Synthesis of sulfanylidene-diazaspirocycloalkanones in a three-component Mannich-type reaction catalyzed with lactic acid
}

\author{
Tadeusz S. Jagodziński,* Jacek G. Sośnicki, and Łukasz Struk
}

West Pomeranian University of Technology Szczecin, Faculty of Chemical Technology and Engineering, Department of Organic and Physical Chemistry, Aleja Piastow 42, 71-065 Szczecin, Poland

E-mail: jagszcz@zut.edu.pl

Received 04-11-2017

Accepted 06-08-2017

Published on line 07-01-2017

\section{Abstract}

The aminomethylation of thioamides containing an acidic hydrogen atom at the $\alpha$-carbon atom was investigated as a general reaction. Spirohexahydropyrimidines were obtained in the one-pot, three-component reaction of a variety of cyclic-ketone-derived thioamides, primary amines and formaldehyde with lactic acid acting as a catalyst. The results of our experiments confirmed the high catalytic efficiency of lactic acid. A proper choice of the starting thioamides and amines makes it possible to use this reaction methodolgy to synthesize several new and potentially useful derivatives of hexahydropyrimidine, e.g., biologically-active compounds.

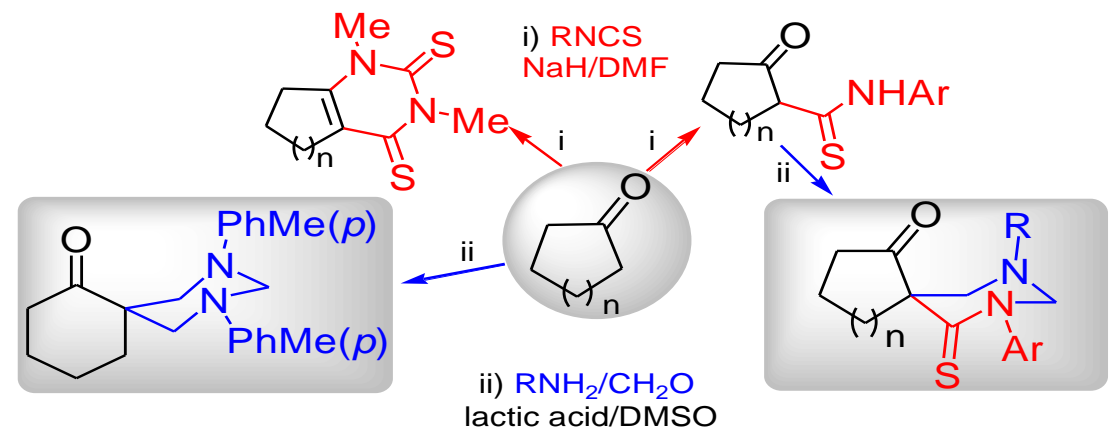

Keywords: Mannich reaction, multicomponent reaction, thioamides, spirohexahydropyrimidines, lactic acid 


\section{Introduction}

Multicomponent reactions (MCRs) have recently attracted the attention of organic chemists since they offer many advantages over the conventional multi-step syntheses. ${ }^{1-4}$ The Mannich-type reaction, a powerful C-C bond-formation process widely applied to the preparation of diverse aminoalkyl derivatives, ${ }^{5-8}$ is often used in MCRs syntheses. Thus, Mukhopadhyay and co-workers reported a multicomponent synthesis of 1,3diarylhexahydropyrimidines in the one-pot iron(III) chloride-catalyzed reaction of 1,3-dicarbonyl compounds, aromatic amines and formaldehyde. ${ }^{9}$ Liang and co-workers lately synthesized 1,3-diaryl-5-spirohexahydropyrimidines via the proline-catalyzed one-pot condensation of anilines, formaldehyde, and cyclohexanones. ${ }^{10}$ Dandia and co-workers obtained several fluorine-containing spirohexahydropyrimidine derivatives using the magnetically separable and easy recyclable heterogeneous $\mathrm{CuFe}_{2} \mathrm{O}_{4}$ nanocatalyst ${ }_{1}^{11}$ while Zeba and co-workers used a chitosan supported Dy(III) catalyst for the fairly green and energy-sustainable synthesis of hexahydropyrimidine derivatives. ${ }^{12} \mathrm{An}$ extensive overview of the recent asymmetric domino reactions has been presented by Pellissier. ${ }^{4}$

Spirocyclic compounds hold a peculiar and enduring fascination for organic chemists. The creation of the stereogenic quaternary $\mathrm{C}$ centers is a challenging task since they are able to generate an asymmetric system with distinct groups attached, and thus open the conditions to research in the fields of asymmetric synthesis, molecular recognition and catalysis. ${ }^{13}$ Many spirocyclic compounds have been isolated from plant and animal sources. ${ }^{14,15}$ The tetrahedral nature of the spiro-linked carbon atom with its specific conformational features and structural implications are of particular value for biological systems. Many spiro heterocycles have exhibited diversified biological activity as well as pharmacological and therapeutic properties. ${ }^{13}$

\section{Results and Discussion}

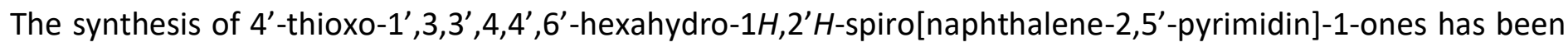
described by us earlier. ${ }^{16}$ The one-pot, three-component reaction (MCRs) of the tetralone-derived thioamides with primary aliphatic amines and formaldehyde was carried out with alcoholic hydrogen chloride as the catalyst. Continuing our studies on the application of thioamides to the synthesis of heterocyclic compounds, ${ }^{16-}$ ${ }^{18}$ we report now on an easy and efficient synthesis of spirohexapyrimidine derivatives from the thioamidefunction-containing cyclic ketones, primary amines and formaldehyde in DMSO in a Mannich-type reaction with lactic acid used as the catalyst. As shown in Scheme 1, the starting thioamides 2a-f were obtained in moderate yields in the reaction of cyclic ketones $1 \mathbf{a}-\mathbf{d}$ with sodium hydride and aryl isothiocyanates in a DMF solution. The analogous reaction of ketones 1 with methyl isothiocyanate yielded bicyclic derivatives of pyrimidine2,4(1H,3H)-dithione 3a-c instead of the expected $\mathrm{N}$-methylthioamide. Cyclopentanone thioanilide $\mathbf{2} \mathbf{g}(\mathrm{n}=1, \mathrm{Ar}$ $=\mathrm{Ph}$ ) was prepared in the reaction of the enamine derivative of the cyclopentanone with phenyl isothiocyanate as reported earlier. ${ }^{19}$ In order to optimize the conditions of the reaction of thioamides $\mathbf{2 a}$ - $\mathrm{g}$ with aliphatic amines and formaldehyde, we concerned ourselves with changing the reactant ratio as well as selecting the most appropriate solvent and catalyst.

With its $\mathrm{p} K_{\mathrm{a}}$ value being one unit lower than that of acetic acid, lactic acid deprotonates ten times more easily. It should, therefore, be a better catalyst in the investigated reactions, the more so that the effects of efficient protonation may be in some way enhanced by the presence of the weakly basic anion. The results of our experiments confirmed the high catalytic efficiency of lactic acid with the added benefit that it is a readily available, low-priced reagent. 
<smiles>Cn1c2c(c(=S)n(C)c1=S)[Te]CC2</smiles>

$3 a-c$

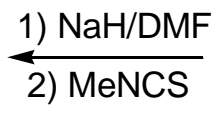

$\mathrm{n}=2,3,4,8$

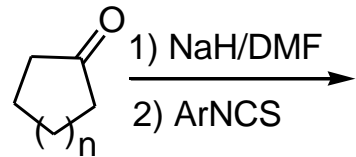

$1 \mathrm{a}-\mathrm{d}$<smiles>O=C1CCCC1C(=S)N[Al]</smiles>

$2 a-f$ 2e: $\mathrm{n}=8, \mathrm{Ar}=\mathrm{Ph}(63 \%)$

2f: $\mathrm{n}=8, \mathrm{Ar}=$ naphthalen-1-yl $(65 \%)$

3a: $n=2(44 \%)$

$3 b: n=4(52 \%)$

$3 c: n=8(58 \%)$

Scheme 1. Preparation of the thioamides 2 and pyrimido-2,4-dithione derivatives 3.

The reactions of thioamides 2a-g with aliphatic amines and formaldehyde in a DMSO solution, and in the presence of lactic acid, were fast and proceeded with high yields without formation of any detectable sideproducts. TLC monitoring showed the reactions to be completed within 2-3 hours (Table 1). Although in the investigated reactions DMSO proves to be the most universal solvent, thioamides react with aliphatic amines and formaldehyde also in an ethanol solution. In some cases, depending on the solubility and ease of the crystallization of the product, the use of ethanol may offer a practical advantage since the crystals that slowly deposit as the reaction proceeds are of excellent purity. Detailed results of the reactions using ethanol are presented in the Experimental Section.

Table 1. Synthesis of sulfanylidene-diazaspirocycloalkanones 4<smiles>CC(=S)C1CCCC1=O</smiles>

$2 a-g$
$\stackrel{\mathrm{N}}{\mathrm{H}}-\mathrm{Ar}+2 \mathrm{CH}_{2} \mathrm{O}+1 \mathrm{RNH}_{2} \underset{\text { room temperature }}{\stackrel{\text { lactic acid } / \mathrm{DMSO}}{\longrightarrow}}$

$n=2,3,4,8$

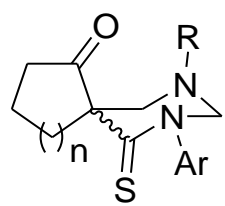

$4 a-I$ (54 - 95\%)

Entry Shioamide 2


Table 1. Continued

\begin{tabular}{|c|c|c|c|c|}
\hline Entry & Thioamide 2 & $\mathrm{RNH}_{2}$ & Spiro compound $4^{a}$ & Yield $(\%)^{b}$ \\
\hline 3 & $2 g$ & & 4c & 54 \\
\hline 4 & $2 a$ & & & 82 \\
\hline 5 & $2 b$ & & $4 \mathrm{H}_{4} \mathrm{Cl}-4$ & 61 \\
\hline 6 & $2 b$ & & $\begin{array}{l}\mathrm{H}_{4} \mathrm{Cl}-4 \\
\mathbf{4 f}\end{array}$ & 72 \\
\hline 7 & 2c & & $4 g$ & 94 \\
\hline 8 & $2 d$ & & $4 \mathrm{Me}-4$ & $\begin{array}{c}95 \\
(84)\end{array}$ \\
\hline 9 & $2 d$ & & $\mathrm{Me}-4$ & 87 \\
\hline
\end{tabular}


Table 1. Continued

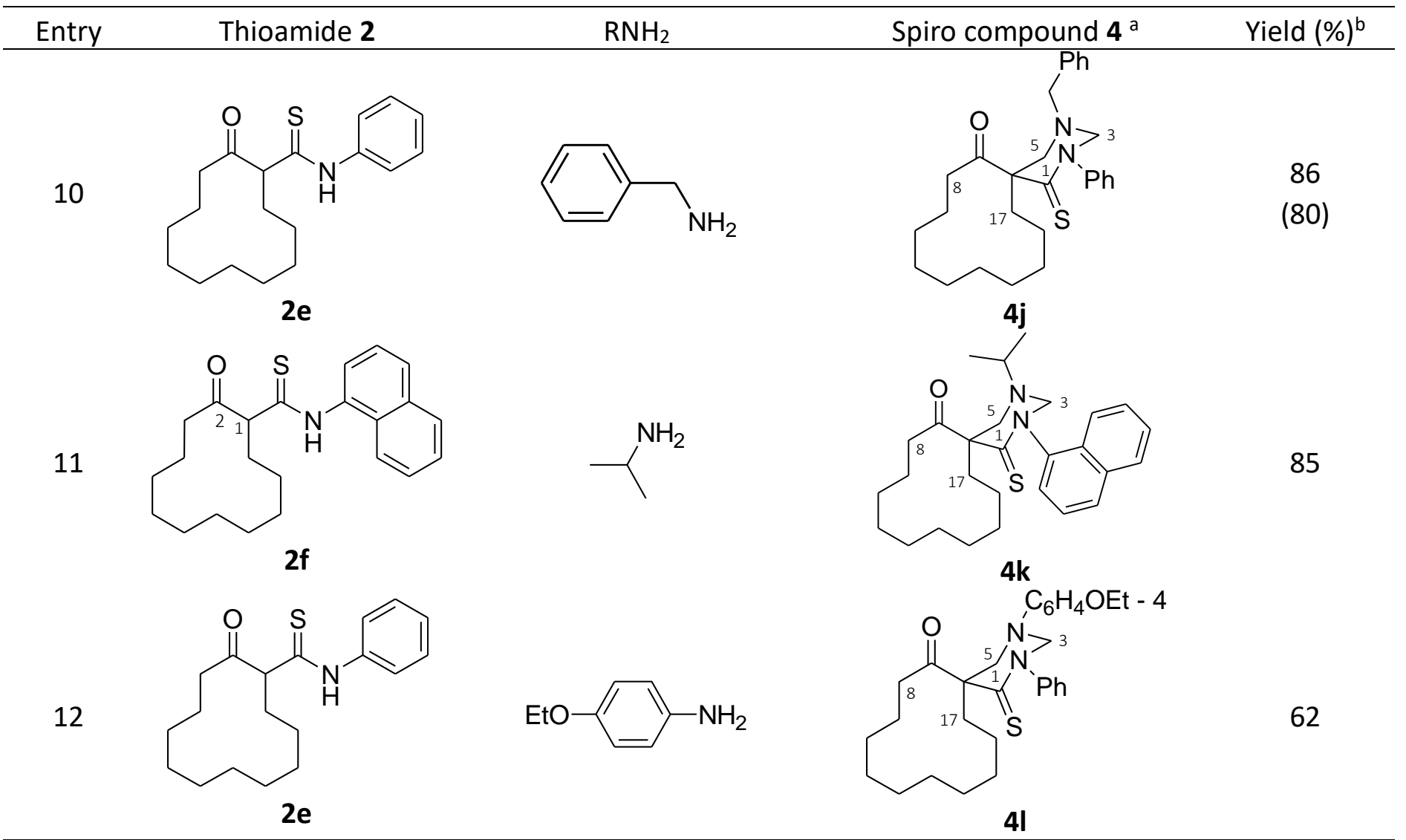

${ }^{a}$ Atom numbering followed in the NMR spectra analyses; ${ }^{b}$ yield of the reaction in DMSO (or EtOH) calculated for the product after column chromatography

In order to get information on whether the structure of lactic acid may affect the enantioselective course of the reactions, L-(+)- and racemic lactic acids were used in parallel runs; the catalyst amounts ranged from a few drops to a multi-fold molar excess. The results failed to reveal any such effect; in all experiments, only racemic derivatives of sulfanylidenediazaspirocycloalkanones (4a-I) were obtained.

In the earlier studies of the three-component reactions of amines and formaldehyde with $\beta$-diketones, $\beta$ keto esters ${ }^{9}$ or cyclohexanone, ${ }^{11,20}$ double aminomethylation was observed to occur at the $\alpha$-carbon atom. Six molecules of reactants were involved and six new covalent bonds were formed in this one-pot, threecomponent reaction. The highest yields of hexahydropyrimidines were obtained when the 1,3-carbonyl compound : amine : formaldehyde molar ratio was $1: 2: 3$. An analogous reaction course was reported in the case of the proline-catalyzed aminomethylation of cyclohexanone. ${ }^{10}$ Our results indicate that the thioamide group nitrogen atom takes part in the formation of the pyrimidine ring. Furthermore, the molar ratio of reagents was widely different, since thioamides $\mathbf{2 a - g}$ reacted with one molecule of the amine and two molecules of formaldehyde. Our suggested mechanistic explanation of this reaction presumes that the electrophilic imine attacks the enol form of the thioamide at both sides to produce two enantiomeric aminothioamides, which subsequently condense with another molecule of formaldehyde to ultimately yield the racemic mixtures of spirocyclic compounds $4 a-I$ (Scheme 2). 


$$
\mathrm{HCHO}+\mathrm{RNH}_{2} \stackrel{+\mathrm{H}^{\oplus} /-\mathrm{H}_{2} \mathrm{O}}{=} \stackrel{\oplus}{=} \stackrel{-=}{=} \mathrm{NR}
$$

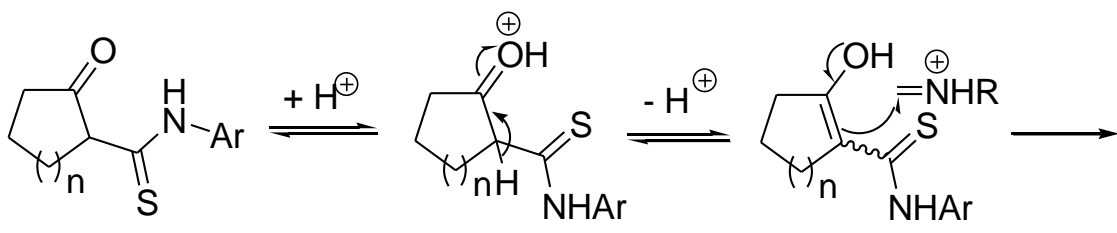

$2 a-g$

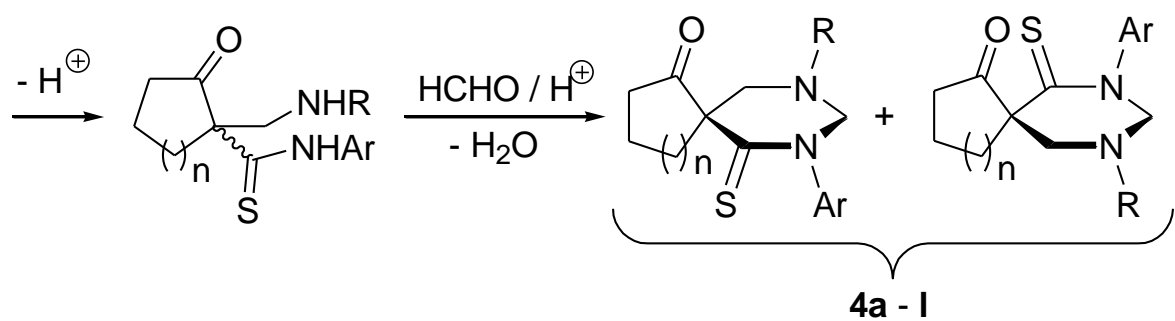

Scheme 2. Proposed mechanism of the three-component Mannich-type reaction catalyzed by lactic acid.

The reaction yield was roughly independent of the substituent attached to the thioamide nitrogen atom. Thioamides of certain cyclic ketones were found to react also with aromatic amines. For instance, the thioamide derived from cyclopentanone $(\mathbf{2 g}, \mathrm{n}=1)$, cyclododecanone $(\mathbf{2 e}, \mathrm{n}=\mathbf{8})$ or cyclohexanone $(\mathbf{2 b}, \mathrm{n}=2)$ reacted with 4-ethoxyaniline or 4-methylaniline to yield spiro compounds $\mathbf{4 c}$, $\mathbf{4 I}$ and $\mathbf{4 f}$, respectively. However, presumably owing to steric hindrance, no such reactions were observed with tetralone-derived thioamides. ${ }^{16}$

The advantages offered by lactic-acid catalysis prompted us to make some preliminary experiments of its use with compounds other than thioamides, and, thus, to find out if it has a more universal applicability to the Mannich-type MCRs. Cyclohexanone was selected as the model reagent since its reactions with aromatic amines and formaldehyde have been studied earlier by numerous authors ${ }^{9-12,20}$ in investigations using both typical and unusual catalysts. The reactions with $p$-tolylamine, $p$-bromoaniline, and $p$-nitroaniline were carried out by us at room temperature in a DMSO solution and, in the case of $p$-tolylamine, also in ethanol.

Preliminary experiments revealed an interesting dependence on the nature of the $p$-substituent. Thus, the reaction with the amine of highest basicity, i.e., $p$-tolylamine, took a typical course to yield 1,3-diaryl-5spirohexahydropyrimidine (5). Cyclohexanone was, however, unreactive when treated with amines containing substituents which decreased their basicity. Depending on the magnitude of the decreasing effect, the reactions yielded either 1,3,5-triazinane derivative (6) or diamine (7). Aliphatic amines did not react under the conditions investigated (Scheme 3). Further research on the reactions using lactic acid as the catalyst is in progress, and the results will be published in due course. 


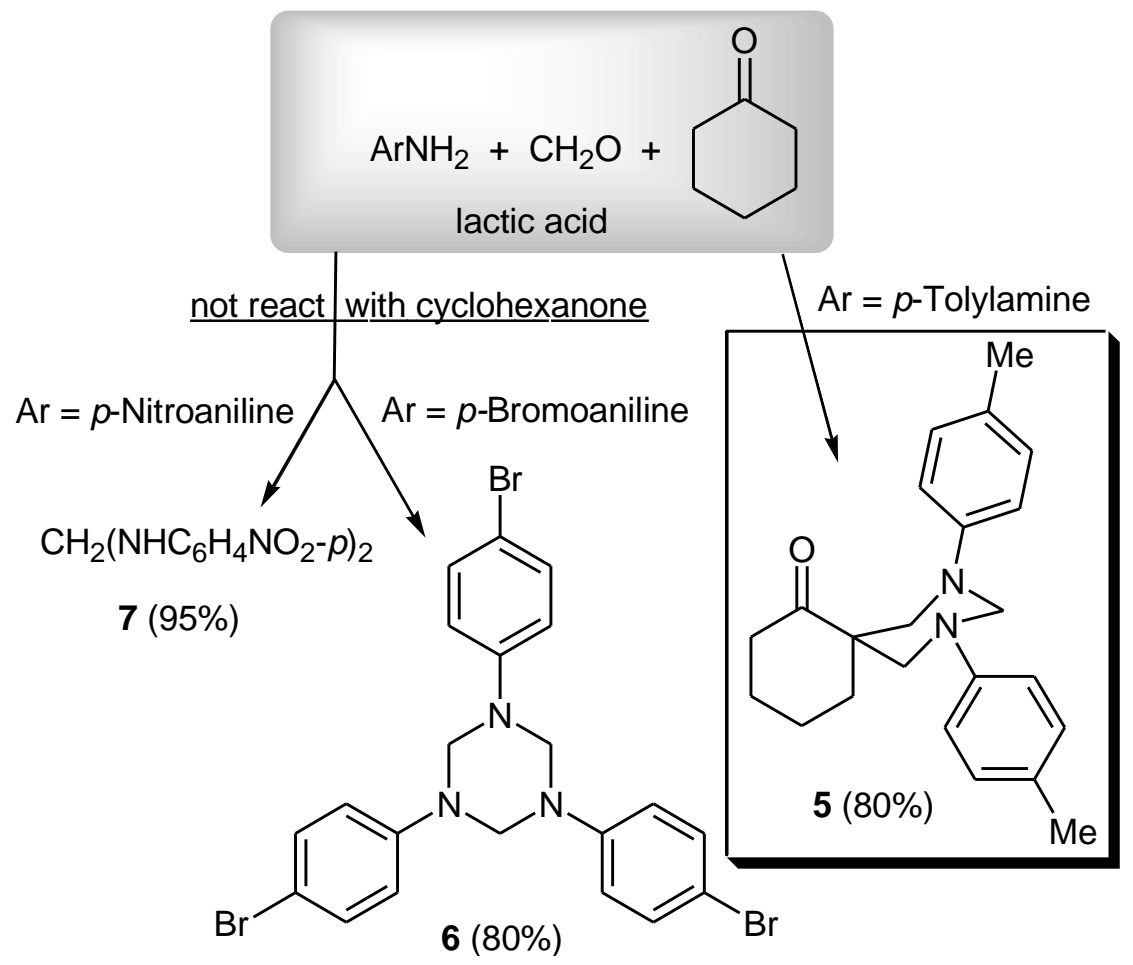

Scheme 3. Preliminary experiments for the six-molecule, three-component reaction catalyzed with lactic acid.

\section{Conclusions}

Aminomethylation of thioamides containing an acidic hydrogen atom at the $\alpha$ carbon atom has to be considered as a general reaction. Its substrates are easily available and the products are formed at room temperature in usually high yields. The results of our experiments confirmed the high catalytic efficiency of lactic acid. A proper choice of the starting thioamides and amines makes it possible to use this reaction to synthesize several new and potentially useful derivatives of hexahydropyrimidine, for example, biologically-active compounds.

\section{Experimental Section}

General. For all synthesized compounds, yields were calculated for the product after column chromatography. Melting points were determined on a Boetius hot-stage apparatus. ${ }^{1} \mathrm{H}$ and ${ }^{13} \mathrm{C}$ NMR spectroscopic measurements were performed on a Bruker DPX $400 \mathrm{HD}$ spectrometer equipped with a $5 \mathrm{~mm} 1 \mathrm{H} / \mathrm{BB}$ probe head, operating at 400.1 and $100.6 \mathrm{MHz}$, respectively. TMS was used as the internal reference. For resolving of multiplets in ${ }^{1} \mathrm{H}$ NMR spectra, ACD/SpecManager program (version 12.01) was used. For detailed peak assignments, 2D spectra were acquired using standard Bruker software. The standard abbreviations for multiplicities were used ( $\mathrm{s}=$ singlet, $\mathrm{d}=$ doublet, $\mathrm{t}=$ triplet, $\mathrm{q}=$ quartet, quint $=$ quintet, $\mathrm{m}=$ multiplet, $\mathrm{sxt}=$ sextet, spt = septet, etc.). Gas chromatography-mass spectrometry (GC-MS) measurements were carried out on an Agilent 7820A GC system equipped with a mass detector Agilent 5977E MSD. Infrared spectra were taken with an Alfa spectrometer with ATR-adapter (Bruker). Elemental analyses were performed on an EuroEA 3000 series, EuroVector CHNS-O Elemental Analyzer. All compounds gave satisfactory elemental-analysis results for $\mathrm{C}, \mathrm{H}, \mathrm{N}$, and S. Specific rotations were measured on a Rudolph Autopol IV digital polarimeter at the wave length 
of $589 \mathrm{~nm}$ at $20{ }^{\circ} \mathrm{C}$ in a $0.5 \%(\mathrm{~m} / \mathrm{V})$ solution in methanol. All reagents (cyclic ketones, amines, isothiocyanates and DMSO) were obtained from Sigma-Aldrich, and used as received. DMF was dried over 4A molecular sieves.

\section{General procedure for the synthesis of thioamides (2a-f) and pyrimidine-2,4(1H,3H)-dithiones (3a-c)}

To a vigorously stirred suspension of sodium hydride $(1 \mathrm{~g}, 60 \%$ dispersion in mineral oil, Sigma-Aldrich) in $25 \mathrm{~mL}$ of dry DMF under argon at room temperature, the appropriate cyclic ketone $(20 \mathrm{mmol})$ was added. When gas evolution terminated $(\sim 3 \mathrm{~h})$, the reaction mixture was cooled to $-10{ }^{\circ} \mathrm{C}$ and a solution of the appropriate aryl or methyl isothiocyanate $(20 \mathrm{mmol})$ in $5 \mathrm{~mL}$ of dry DMF was added slowly to keep the temperature $<0{ }^{\circ} \mathrm{C}$. The mixture was stirred for 1 hour at $0-5^{\circ} \mathrm{C}$ and then 1 hour at room temperature, left in a refrigerator overnight, and then poured onto crushed ice and acidified with $2 \mathrm{M} \mathrm{HCl}$. After $3 \mathrm{~h}$, most of the water-DMF solution was decanted. The oily residue was dissolved in $\mathrm{CH}_{2} \mathrm{Cl}_{2}$, washed twice with $1 \mathrm{M} \mathrm{HCl}$ and water, then evaporated and purified by chromatography on silica gel using $\mathrm{CH}_{2} \mathrm{Cl}_{2}$ as the eluent. Concentration of the eluates under reduced pressure gave products which were finally purified by recrystallization from heptane/toluene = 2:1.

2-Oxo- $N$-phenylcyclohexane-1-carbothioamide (2a). White fluffy crystals; yield: $66 \%$; $\mathrm{mp} 79-81{ }^{\circ} \mathrm{C}$ (from heptane/toluene = 2:1). Lit. ${ }^{19} \mathrm{mp} 97{ }^{\circ} \mathrm{C}$; Lit. ${ }^{21} \mathrm{mp} 84-89{ }^{\circ} \mathrm{C}$. IR $\left(\mathrm{ATR}, \mathrm{V}_{\max } \mathrm{cm}^{-1}\right)$ : $3312(\mathrm{NH}) ; 3136,3053,2941$, 2918, $2871(\mathrm{CH}) ; 1692(\mathrm{CO})$. Anal. Calcd for $\mathrm{C}_{13} \mathrm{H}_{15} \mathrm{NOS}$ : C, 66.92; $\mathrm{H}, 6.48 ; \mathrm{N}, 6.00 ; \mathrm{S}$ 13.74. Found: C, 66.66; $\mathrm{H}$, $6.81 ; \mathrm{N}, 5.98 ; \mathrm{S} 13.68 \%$.

$\mathbf{N}$-(4-chlorophenyl)-2-oxocyclohexane-1-carbothioamide (2b). Pale yellow crystals; yield: $55 \%$; mp $102-104{ }^{\circ} \mathrm{C}$ (from EtOH); Lit. ${ }^{22} \mathrm{mp} 98-101{ }^{\circ} \mathrm{C}$. IR (ATR, $\mathrm{V}_{\max } \mathrm{cm}^{-1}$ ): 3308, 3273 (NH); 2946, 2932, 2863 (CH); 1695 (CO). Anal. Calcd for $\mathrm{C}_{13} \mathrm{H}_{14} \mathrm{CINOS:} \mathrm{C,} \mathrm{58.32;} \mathrm{H,} \mathrm{5.27;} \mathrm{N,} \mathrm{5.23;} \mathrm{S,} \mathrm{11.97.} \mathrm{Found:} \mathrm{C,} \mathrm{58.46;} \mathrm{H,} \mathrm{5.48;} \mathrm{N,} \mathrm{5.28;} \mathrm{S,} 12.17 \%$.

2-Oxo-N-phenylcycloheptane-1-carbothioamide (2c). Pale yellow crystals; yield: $59 \%$; $\mathrm{mp} 93-95{ }^{\circ} \mathrm{C}$ (from heptane/toluene 2:1); Lit. ${ }^{19} \mathrm{mp} 93.5^{\circ} \mathrm{C}$. IR (ATR, $\mathrm{V}_{\max } \mathrm{Cm}^{-1}$ ): 3242 (NH); 3034, 2936, 2920, 2855 (CH); 1689 (CO). $\mathbf{N}$-(4-Methylphenyl)-2-oxocyclooctane-1-carbothioamide (2d). Pale yellow crystals; yield $73 \%$; $\mathrm{mp} 78-80{ }^{\circ} \mathrm{C}$ (from hexane). IR (ATR, $V_{\max } \mathrm{Cm}^{-1}$ ): 3269 (NH); 3033, 2989, 2971, 2934, 2918, 2855 (CH); 1683 (CO). ${ }^{1} \mathrm{H}$ NMR (400 $\left.\mathrm{MHz} \mathrm{CDCl}_{3}, 23^{\circ} \mathrm{C}\right): \delta=1.11-1.23(\mathrm{~m}, 1 \mathrm{H}, \underline{\mathrm{CH}} \mathrm{H}), 1.50-1.93\left(\mathrm{~m}, 6 \mathrm{H}, 3 \times \mathrm{CH}_{2}\right), 2.00(\mathrm{dtd}, 1 \mathrm{H}, J 13.0,12.0,3.2 \mathrm{~Hz}$,

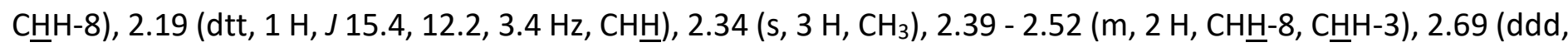
$1 \mathrm{H}, J$ 15.0, 6.5, 3.2 Hz, CHㅍ-3), 4.51 (dd, $1 \mathrm{H}, J$ 12.0, $3.2 \mathrm{~Hz}, \mathrm{CH}-1), 7.19$ (d, $2 \mathrm{H}, J 8.3 \mathrm{~Hz}, \mathrm{ArH}), 7.58$ (d, $2 \mathrm{H}, J 8.3$ $\mathrm{Hz}, \mathrm{ArH}), 10.75$ (br. s., $1 \mathrm{H}, \mathrm{NH})$ ppm. ${ }^{13} \mathrm{C} \mathrm{NMR}\left(100 \mathrm{MHz}, \mathrm{CDCl}_{3}, 23^{\circ} \mathrm{C}\right): \delta=21.14\left(\mathrm{CH}_{3}\right), 22.48,24.57,25.36,28.44$ $\left.\left(4 \times \mathrm{CH}_{2}\right), 40.80\left(\mathrm{CH}_{2}-8\right), \mathrm{CH}_{2}-3\right), 65.32(\mathrm{CH}-1), 123.31,129.38,136.30,136.68\left(\mathrm{C}_{6} \mathrm{H}_{4}\right), 198.24(\mathrm{C}=\mathrm{S}), 218.92(\mathrm{C}=\mathrm{O})$ ppm. Anal. Calcd for $\mathrm{C}_{16} \mathrm{H}_{21} \mathrm{NOS}$ : C, 69.78; H, 7.69; N, 5.09; S, 11.64. Found: $\mathrm{C}, 69.55 ; \mathrm{H}, 7.90 ; \mathrm{N}, 5.00 ; \mathrm{S} 11.78 \%$. 2-Oxo- $\mathbf{N}$-phenylcyclododecane-1-carbothioamide (2e). Pale yellow crystals; yield: $63 \%$; mp $123-125{ }^{\circ} \mathrm{C}$ (from heptane/toluene 2:1). Lit. ${ }^{21} \mathrm{mp} 123-124{ }^{\circ} \mathrm{C} \mathrm{IR}\left(\mathrm{ATR}, \mathrm{v}_{\max } \mathrm{cm}^{-1}\right)$ : 3295 (NH); 2927, 2903, 2861 (CH); 1700 (CO).

${ }^{1} \mathrm{H}$ NMR $\left(400 \mathrm{MHz} \mathrm{CDCl}_{3}, 23^{\circ} \mathrm{C}\right): \delta=1.13-1.45\left(\mathrm{~m}, 14 \mathrm{H}, 7 \times \mathrm{CH}_{2}\right), 1.48-1.62(\mathrm{~m}, 1 \mathrm{H}, \mathrm{CH} \mathrm{H}), 1.95-2.05(\mathrm{~m}, 1 \mathrm{H}$, CㅂH), $2.11-2.20(\mathrm{~m}, 1 \mathrm{H}, \mathrm{C} \underline{\mathrm{H}} \mathrm{H}-3), 2.27-2.37$ (m, $1 \mathrm{H}, \mathrm{CH} \underline{H}-3), 2.46$ (ddd, $1 \mathrm{H}, \mathrm{J}=17.6,6.6,3.8 \mathrm{~Hz}, \mathrm{C} \underline{H} \mathrm{H}-12), 3.01$ (ddd, $1 \mathrm{H}, \mathrm{J}=17.6,11.0,3.2 \mathrm{~Hz}, \mathrm{CH} \underline{\mathrm{H}}-12), 4.38$ (dd, $1 \mathrm{H}, \mathrm{J}=7.9,3.8 \mathrm{~Hz}, \mathrm{CH}-1), 7.23-7.28(\mathrm{~m}, 1 \mathrm{H}, \mathrm{C} 6 \mathrm{H} 5), 7.37-7.43$ (m, $2 \mathrm{H}, \mathrm{C} 6 \mathrm{H} 5), 7.71-7.77$ (m, 2H, C6H5), 10.92 (br. s., $1 \mathrm{H}, \mathrm{NH})$ ppm. ${ }^{13} \mathrm{C} \mathrm{NMR}\left(100 \mathrm{MHz}, \mathrm{CDCl}_{3}, 23^{\circ} \mathrm{C}\right): \delta=21.62$, 21.85, 21.98, 23.50, 23.73, 23.97, 25.56, $25.71\left(8 \times \mathrm{CH}_{2}\right), 32.93\left(\mathrm{CH}_{2}-3\right), 40.42\left(\mathrm{CH}_{2}-12\right), 65.96(\mathrm{CH}-1), 123.31$, 126.82, 128.85, $138.62\left(\mathrm{C}_{6} \mathrm{H}_{5}\right), 198.49(\mathrm{C}=\mathrm{S}), 213.93(\mathrm{C}=\mathrm{O}) \mathrm{ppm}$. Anal. Calcd for $\mathrm{C}_{19} \mathrm{H}_{27} \mathrm{NOS}$ : C, 71.88; $\mathrm{H}, 8.57 ; \mathrm{N}$, 4.41; S, 10.10. Found: C, 71.92; H, 8.94; N, 4.36; S, 10.19\%.

$\mathbf{N}$-(Naphthalen-1-yl)-2-oxocyclododecane-1-carbothioamide (2f). Pale yellow crystals; yield: 65\%; mp 171-173 ${ }^{\circ} \mathrm{C}$ (from heptane/toluene 2:1). IR (ATR, $\mathrm{V}_{\max } \mathrm{cm}^{-1}$ ): $3303(\mathrm{NH}) ; 2927,2899,2890,2839(\mathrm{CH}) ; 1699$ (CO). ${ }^{1} \mathrm{H} N M R$ $\left(400 \mathrm{MHz}^{\mathrm{CDCl}} 3,2{ }^{\circ} \mathrm{C}\right): \delta={ }^{1} \mathrm{H}$ NMR $(400 \mathrm{MHz}): \delta=1.16-1.64\left(\mathrm{~m}, 15 \mathrm{H}, \mathrm{C} \underline{\mathrm{H}}, 7 \times \mathrm{CH}_{2}\right), 2.01-2.12(\mathrm{~m}, 1 \mathrm{H}$, $\mathrm{CH} \underline{H}), 2.22-2.32(\mathrm{~m}, 1 \mathrm{H}, \mathrm{C} \underline{\mathrm{H}} \mathrm{H}-3), 2.4--2.50(\mathrm{~m}, 1 \mathrm{H}, \mathrm{CH} \underline{\mathrm{H}}-3), 2.55$ (ddd, $1 \mathrm{H}, \mathrm{J}=17.6,6.6,3.4 \mathrm{~Hz}, \mathrm{C} \underline{\mathrm{H}} \mathrm{H}-12), 3.11$ (ddd, $1 \mathrm{H}, \mathrm{J}=17.6,10.7,3.2 \mathrm{~Hz}, \mathrm{CH} \underline{\mathrm{H}}-12$ ), 4.56 (dd, $1 \mathrm{H}, \mathrm{J}=7.9,3.8 \mathrm{~Hz}, \mathrm{CH}-1), 7.49$ - 7.59 (m, $3 \mathrm{H}, 1-\mathrm{Naph}), 7.80$ - 
7.91 (m, $3 \mathrm{H}, 1-\mathrm{Naph}), 7.96$ (d, $1 \mathrm{H}, \mathrm{J}=7.6 \mathrm{~Hz}, 1-\mathrm{Naph}), 11.07$ (br. s., $1 \mathrm{H}, \mathrm{NH}) \mathrm{ppm} .{ }^{13} \mathrm{C} \mathrm{NMR}\left(100 \mathrm{MHz}, \mathrm{CDCl}_{3}, 23\right.$ $\left.{ }^{\circ} \mathrm{C}\right): \delta=21.70,22.05,22.21,23.61,23.81,24.06,25.62,25.65\left(8 \times \mathrm{CH}_{2}\right), 33.04\left(\mathrm{CH}_{2}-3\right), 40.60\left(\mathrm{CH}_{2}-12\right), 65.75(\mathrm{CH}-$ 1), 121.41, 123.44, 125.19, 126.36, 126.93, 127.92, 128.24, 128.70, 134.21, 134.37 (1-Naph), 200.59, (C=S), 214.04 (C=O) ppm. Anal. Calcd for $\mathrm{C}_{23} \mathrm{H}_{29} \mathrm{NOS}: \mathrm{C}, 75.16 ; \mathrm{H}, 7.95 ; \mathrm{N}, 3.81 ; \mathrm{S}, 8.72$. Found: C, 75.19; $\mathrm{H}, 7.92 ; \mathrm{N}$, 3.74; S 8.63\%.

2-Oxo- $\mathbf{N}$-phenylcyclopentane-1-carbothioamide (2g): prepared from 4-(cyclopent-1-en-1-yl)morpholine and phenyl isothiocyanate as described earlier. ${ }^{19}$ Pale yellow crystals; yield $87 \%$; $\mathrm{mp}$ 99-101 ${ }^{\circ} \mathrm{C}$ (from heptane/toluene 1:1); Lit. ${ }^{21} \mathrm{mp} 96-97^{\circ} \mathrm{C} . \mathrm{IR}\left(\mathrm{ATR}, \mathrm{V}_{\max } \mathrm{cm}^{-1}\right)$ : 3307(NH); 2976, 2954, 2911, 2855,2826 (CH); 1604-1585 (CO).

1,3-Dimethyl-5,6,7,8-tetrahydro-1H-quinazoline-2,4-dithione (3a). Yellow crystals; yield: $44 \%$; $\mathrm{mp} 195-196{ }^{\circ} \mathrm{C}$ (from heptane/toluene 2:1); Lit. ${ }^{23} \mathrm{mp} 185{ }^{\circ} \mathrm{C}$. IR (ATR, $\left.\mathrm{v}_{\max } \mathrm{cm}^{-1}\right): 2942,2859,1588,1430 .{ }^{1} \mathrm{H} \mathrm{NMR}(400 \mathrm{MHz}$, $\left.\mathrm{CDCl}_{3}, 23^{\circ} \mathrm{C}\right): \delta=1.69-1.78\left(\mathrm{~m}, 2 \mathrm{H}, \mathrm{CH}_{2}-6(7)\right), 1.78-1.88\left(\mathrm{~m}, 2 \mathrm{H}, \mathrm{CH}_{2}-7(6)\right), 2.63\left(\mathrm{t}, 2 \mathrm{H}, \mathrm{J}=6.3 \mathrm{~Hz}, \mathrm{CH}_{2}-5(8)\right)$, $2.73\left(\mathrm{t}, 2 \mathrm{H}, \mathrm{J}=6.3 \mathrm{~Hz}, \mathrm{CH}_{2}-8(5)\right), 3.91\left(\mathrm{~s}, 3 \mathrm{H}, \mathrm{NCH}_{3}\right), 4.38\left(\mathrm{~s}, 3 \mathrm{H}, \mathrm{NCH}_{3}\right)$ ppm. ${ }^{13} \mathrm{C} \mathrm{NMR}\left(100 \mathrm{MHz}, \mathrm{CDCl}_{3}, 23^{\circ} \mathrm{C}\right): \delta$ $=21.61,22.13\left(\mathrm{CH}_{2}-6, \mathrm{CH}_{2}-7\right), 28.71,28.85\left(\mathrm{CH}_{2}-5, \mathrm{CH}_{2}-8\right), 39.93,45.02\left(2 \times \mathrm{NCH}_{3}\right), 124.70$ (C-4a), 144.67 (C-8a), 174.73 (C-2), 186.90 (C-4) ppm. MS (El, $70 \mathrm{eV}$ ): m/z (\%) 226 (100) [M]+., 211 (25), 193 (49). Anal. Calcd for $\mathrm{C}_{10} \mathrm{H}_{14} \mathrm{~N}_{2} \mathrm{~S}_{2}$ : C, 53.06; $\mathrm{H}, 6.23 ; \mathrm{N}, 12.38 ; \mathrm{S}, 28.33$. Found: $\mathrm{C}, 53.20 ; \mathrm{H}, 6.38 ; \mathrm{N}, 12.10 ; \mathrm{S}, 28.41 \%$.

1,3-Dimethyl-5,6,7,8,9,10-hexahydrocycloocta[d]pyrimidine-2,4(1H,3H)-dithione (3b). Yellow crystals; yield: 52\%; $m p 137-139{ }^{\circ} \mathrm{C}$ (from heptane/toluene 2 : 1). IR (ATR, $v_{\max } \mathrm{cm}^{-1}$ ): 2930, 2918, 2846, 1579, 1487, 1463, 1435, 1413. ${ }^{1} \mathrm{H}$ NMR (400 MHz, $\left.\mathrm{CDCl}_{3}, 23{ }^{\circ} \mathrm{C}\right): \delta=1.47-1.56\left(\mathrm{~m}, 4 \mathrm{H}, 2 \times \mathrm{CH}_{2}\right), 1.74-1.88\left(\mathrm{~m}, 4 \mathrm{H}, 2 \times \mathrm{CH}_{2}\right), 2.86-2.96$ (m, $2 \mathrm{H}, \mathrm{CH}_{2}$ ), 3.09 (br. s., $\left.2 \mathrm{H}, \mathrm{CH}_{2}\right), 3.97$ (s, $\left.3 \mathrm{H}, \mathrm{NCH}_{3}\right), 4.38\left(\mathrm{~s}, 3 \mathrm{H}, \mathrm{NCH}_{3}\right)$ ppm. ${ }^{13} \mathrm{C} \mathrm{NMR}\left(100 \mathrm{MHz} \mathrm{CDCl}_{3}, 23\right.$ $\left.{ }^{\circ} \mathrm{C}\right): \delta \mathrm{ppm}=26.01,26.96,28.23,28.64,29.75,31.30\left(6 \times \mathrm{CH}_{2}\right), 41.19,45.77\left(\mathrm{CH}_{2}-5, \mathrm{CH}_{2}-10\right), 127.52$ (=C-4a), 146.18 (=C-10a), 175.20 (S=C-2), 186.25 (S=C-4) ppm. MS (El, 70eV): m/z (\% I max $)=254$ (100) [M] ${ }^{+}, 239(30), 225$ (32), 221 (44), 211 (24). Anal. Calcd for $\mathrm{C}_{12} \mathrm{H}_{18} \mathrm{~N}_{2} \mathrm{~S}_{2}$ : C, 56.65; H, 7.13; N, 11.01; S, 25.21. Found: C, 56.69; H, 7.55; N, 10.87; S, 25.56\%.

1,3-Dimethyl-5,6,7,8,9,10,11,12,13,14-decahydrocyclododecapyrimidine-2,4(1H,3H)-dithione (3c). Yellow crystals; yield: $52 \%$; $\mathrm{mp} 205-207{ }^{\circ} \mathrm{C}$ (heptane/toluene 2:1); mp 208-210 ${ }^{\circ} \mathrm{C}$ (from heptane). IR (ATR, $\mathrm{V}_{\max } \mathrm{Cm}^{-1}$ ): 2916, 2846, 1572, 1466, 1421. ${ }^{1} \mathrm{H}$ NMR $\left(400 \mathrm{MHz}, \mathrm{CDCl}_{3}, 23{ }^{\circ} \mathrm{C}\right): \delta=1.39-1.45\left(\mathrm{~m}, 12 \mathrm{H}, 6 \times \mathrm{CH}_{2}\right), 1.69-1.81(\mathrm{~m}$, $4 \mathrm{H}, 2 \times \mathrm{CH}_{2}$ ), 2.80 (br s, $\left.2 \mathrm{H}, \mathrm{CH}_{2}\right), 2.94\left(\mathrm{t}, \mathrm{J}=7.7 \mathrm{~Hz}, 2 \mathrm{H}, \mathrm{CH}_{2}\right), 3.95\left(\mathrm{~s}, 3 \mathrm{H}, \mathrm{NCH}_{3}\right), 4.37\left(\mathrm{~s}, 3 \mathrm{H}, \mathrm{NCH}_{3}\right) \mathrm{ppm}^{13} \mathrm{C}$ $\operatorname{NMR}\left(100 \mathrm{MHz}_{2} \mathrm{CDCl}_{3}, 23^{\circ} \mathrm{C}\right): \delta=21.99,22.20,25.57,25.93,26.59,27.41,27.57,27.77,29.68,30.56,\left(10 \times \mathrm{CH}_{2}\right)$, 41.65, $45.88\left(2 \times \mathrm{CH}_{3}\right.$ ), 128.11 (=C-4a), 146.78 (=C-14a), 175.01 (S=C-2), 187.26 (S=C-4) ppm. MS (EI, 70 ev): $\mathrm{m} / z$ $\left(\% I_{\max }\right)=310(60)[\mathrm{M}]^{+*}, 295$ (12), 277 (100). Anal. Calcd for $\mathrm{C}_{16} \mathrm{H}_{26} \mathrm{~N}_{2} \mathrm{~S}_{2}$ : C, 61.89; H, 8.44; N, 9.02; S, 20.65. Found: C, 61.85; H, 8.764; N, 8.92, S $20.47 \%$.

Synthesis of sulfanylidene-diazaspirocycloalkanones (4a-I) in DMSO (General procedure for the threecomponent reaction). A mixture of thioamide $(1 \mathrm{mmol})$, the appropriate aliphatic or aromatic primary amine (1.3-1.5 mmol), formaldehyde ( $4 \mathrm{mmol}, 37 \%$ aqueous solution), and $0.5 \mathrm{~mL}$ of lactic acid [80\% L-(+)-; or DL-] in $5 \mathrm{~mL}$ DMSO was stirred at room temperature for 2-5 hour. The reaction progress was monitored by TLC $\left(\mathrm{SiO}_{2} /\right.$ chloroform or hexane/ethyl acetate 1:1). After this time, the reaction was quenched by addition of water and some solid $\mathrm{NaHCO}_{3}$. The resulting precipitate was filtered off, washed with water and dried. The crude product was purified by silica gel column chromatography (hexane/EtOAc $=2: 1$ or $\mathrm{CH}_{2} \mathrm{Cl}_{2}$ ). Concentration of the eluate under reduced pressure gave products which were finally purified by recrystallization from an appropriate solvent. 


\section{Some examples of the reaction in EtOH: Synthesis of spiro compounds $4 b, h, j$}

A mixture of thioamide $\mathbf{2} \mathbf{g}, \mathbf{2} \mathbf{d}$ or $\mathbf{2 e}(1 \mathrm{mmol})$, the appropriate primary amine $(1.5 \mathrm{mmol})$, and formaldehyde (4 $\mathrm{mmol}, 36 \%$ aqueous solution) in $10 \mathrm{~mL}$ of EtOH containing 5-10 drops of DL-lactic acid was stirred at room temperature for $1 \mathrm{~h}$ and then left overnight. Next day, the crystalline products were filtered off and recrystallized from ethanol.

9-Benzyl-7-phenyl-6-sulfanylidene-7,9-diazaspiro[4,5]decane-1-one (4a). Yellowish semi-solid; yield: 92\%. IR (ATR, $\left.v_{\max } \mathrm{cm}^{-1}\right)$ : 3060, 3029, 2964, $2812(\mathrm{CH}) ; 1735$ (CO). ${ }^{1} \mathrm{H}$ NMR $\left(400 \mathrm{MHz}, \mathrm{CDCl}_{3}, 23^{\circ} \mathrm{C}\right): \delta=1.68-1.81(\mathrm{~m}, 1$ $\mathrm{H}, \underline{\mathrm{C}} \underline{\mathrm{H}} \mathrm{H}-3), 2.23$ - 2.36 (m, $3 \mathrm{H}, \mathrm{CH} \underline{\mathrm{H}}-3, \mathrm{C} \underline{\mathrm{H}} \mathrm{H}-4, \mathrm{C} \underline{\mathrm{H}} \mathrm{H}-2), 2.70(\mathrm{dt}, 1 \mathrm{H}, J 18.8,9.4 \mathrm{~Hz}, \mathrm{CH} \underline{\mathrm{H}}-2), 2.92(\mathrm{~d}, 1 \mathrm{H}, J 12.6 \mathrm{~Hz}$,

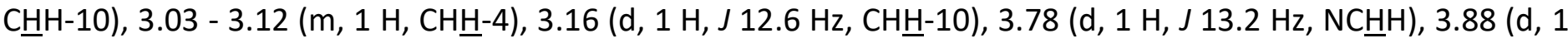
$\mathrm{H}, J 13.2 \mathrm{~Hz}, \mathrm{NCH} \underline{\mathrm{H}}), 4.37$ (s, $\left.2 \mathrm{H}, \mathrm{CH}_{2}-8\right), 7.19$ - $7.24\left(\mathrm{~m}, 2 \mathrm{H}, \mathrm{C}_{6} \mathrm{H}_{5}\right), 7.27-7.40\left(\mathrm{~m}, 6 \mathrm{H}, 2 \times \mathrm{C}_{6} \mathrm{H}_{5}\right), 7.42$ - 7.48 (m, $\left.2 \mathrm{H}, \mathrm{C}_{6} \mathrm{H}_{5}\right)$ ppm. ${ }^{13} \mathrm{C}$ NMR $\left(100 \mathrm{MHz}, \mathrm{CDCl}_{3}, 23^{\circ} \mathrm{C}\right): \delta \mathrm{ppm}=19.88\left(\mathrm{CH}_{2}-3\right), 39.21\left(\mathrm{CH}_{2}-2\right), 40.98\left(\mathrm{CH}_{2}-4\right), 56.08$ $\left(\mathrm{CH}_{2}-10\right), 57.91\left(\mathrm{NCH}_{2}\right), 61.58(\mathrm{C}-6), 74.28\left(\mathrm{CH}_{2}-8\right), 126.88,127.88,128.31,128.68,128.74,129.88,136.46$, 144.22 ( $\left.2 \times \mathrm{C}_{6} \mathrm{H}_{5}\right) 202.39$ (C=S), 215.93 (C=O) ppm. Anal. Calcd for $\mathrm{C}_{21} \mathrm{H}_{22} \mathrm{~N}_{2} \mathrm{OS}: \mathrm{C} 71.97 ; \mathrm{H}, 6.33 ; \mathrm{N}, 7.99 ; \mathrm{S}, 8.43$. Found: $\mathrm{C}, 71.84 ; \mathrm{H}, 6.68 ; \mathrm{N}, 7.95 ; \mathrm{S}, 8.63 \%$.

9-(4-Chlorobenzyl)-7-phenyl-6-sulfanylidene-7,9-diazaspiro[4,5]decane-1-one (4b). White crystals; yield: 87\% (DMSO); 82\% (EtOH); mp 120-122 ${ }^{\circ} \mathrm{C}$ (from EtOH). IR (ATR, $\mathrm{V}_{\max } \mathrm{cm}^{-1}$ ): 3050, 2964, 2827 (CH); 1735 (CO). ${ }^{1} \mathrm{H}$ NMR $\left(400 \mathrm{MHz}, \mathrm{CDCl}_{3}, 23^{\circ} \mathrm{C}\right): \delta=1.70-1.83(\mathrm{~m}, 1 \mathrm{H}, \underline{\mathrm{C}} \underline{\mathrm{H}} \mathrm{H}-3), 2.22-2.35(\mathrm{~m}, 3 \mathrm{H}, \mathrm{C} \underline{\mathrm{H}} \mathrm{H}-2, \mathrm{CH} \underline{\mathrm{H}}-3, \mathrm{C} \underline{\mathrm{H}}-4), 2.70(\mathrm{dt}, 1$ $\mathrm{H}, J$ 19.0, 9.7 Hz, CHㅡ-2), 2.90 (d, $1 \mathrm{H}, J 12.7 \mathrm{~Hz}, \underline{\mathrm{C}} \underline{\mathrm{H}}-10), 3.03-3.12(\mathrm{~m}, 1 \mathrm{H}, \mathrm{CH} \underline{\mathrm{H}}-4), 3.16$ (d, $1 \mathrm{H}, J 12.7 \mathrm{~Hz}$, $\mathrm{CH} \underline{\mathrm{H}}-10), 3.77$ (d, $1 \mathrm{H}, \mathrm{J} 13.4 \mathrm{~Hz}, \mathrm{NC} \underline{\mathrm{H}}$ ), 3.84 (d, $1 \mathrm{H}, \mathrm{J} 13.4 \mathrm{~Hz}, \mathrm{NCH} \underline{\mathrm{H}}), 4.36\left(\mathrm{~s}, 2 \mathrm{H}, \mathrm{CH}_{2}-8\right), 7.18-7.23(\mathrm{~m}, 2 \mathrm{H}$, $\mathrm{ArH})$, 7.28-7.39 (m, 5H, $\left.\mathrm{C}_{6} \mathrm{H}_{5}\right), 7.42-7.48(\mathrm{~m}, 2 \mathrm{H}, \mathrm{ArH}) \mathrm{ppm} .{ }^{13} \mathrm{C} \mathrm{NMR}\left(100 \mathrm{MHz}, \mathrm{CDCl}_{3}, 23{ }^{\circ} \mathrm{C}\right): \delta \mathrm{ppm}=19.88$ $\left(\mathrm{CH}_{2}-3\right), 39.12\left(\mathrm{CH}_{2}-2\right), 40.05\left(\mathrm{CH}_{2}-4\right), 56.11\left(\mathrm{CH}_{2}-10\right), 57.11\left(\mathrm{NCH}_{2}\right), 61.45(\mathrm{C}-6), 74.16\left(\mathrm{CH}_{2}-8\right), 126.82,128.37$, 128.86, 129.93, 130.01, 133.64, 135.05, 144.17 (4- $\left.\mathrm{ClC}_{6} \mathrm{H}_{4}, \mathrm{C}_{6} \mathrm{H}_{5}\right) 202.29$ (C=S), 215.80 (C=O) ppm. Anal. Calcd for $\mathrm{C}_{21} \mathrm{H}_{21} \mathrm{ClN}_{2} \mathrm{OS}: \mathrm{C}, 65.53 ; \mathrm{H}, 5.50 ; \mathrm{N}, 7.28 ; \mathrm{S}, 8.33$. Found: $\mathrm{C}, 65.84 ; \mathrm{H}, 5.76 ; \mathrm{N}, 7.27 ; \mathrm{S}, 8.70 \%$.

9-(4-Ethoxyphenyl)-7-phenyl-6-sulfanylidene-7,9-diazaspiro[4,5]decane-1-one (4c). Yellow crystals; yield:

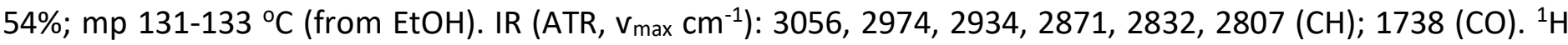
NMR $\left(400 \mathrm{MHz}, \mathrm{CDCl}_{3}, 2{ }^{\circ} \mathrm{C}\right): \delta=1.40\left(\mathrm{t}, J 7.0 \mathrm{~Hz}, 3 \mathrm{H}, \mathrm{CH}_{3}\right), 1.77-1.90(\mathrm{~m}, 1 \mathrm{H}, \mathrm{C} \underline{\mathrm{H}}-3), 2.10-2.19(\mathrm{~m}, 1 \mathrm{H}$, CㅂH), 2.23 - 2.37 (m, $2 \mathrm{H}, \mathrm{CH} \underline{\mathrm{H}}-3, \mathrm{C} \underline{\mathrm{H}}), 2.67-2.84$ (m, $2 \mathrm{H}, 2 \times \mathrm{CH} \underline{\mathrm{H}}), 3.57$ (dd, J 13.2, $1.7 \mathrm{~Hz}, 1 \mathrm{H}, \mathrm{C} \underline{\mathrm{H}}-10), 3.77$ (dd, J 13.2, 0.7 Hz, $1 \mathrm{H}, \mathrm{CH} \underline{\mathrm{H}}-10), 4.00$ (q, J 7.0 Hz, $2 \mathrm{H}, \mathrm{OCH}_{2}$ ), 4.86 (dd, J 11.8, 1.7 Hz, $1 \mathrm{H}, \mathrm{C} \underline{\mathrm{H}}-8$ ), 4.93 (d, J 11.8, $1 \mathrm{H}, \mathrm{CH} \underline{\mathrm{H}}-8), 6.84-6.89(\mathrm{~m}, 2 \mathrm{H}, \mathrm{ArH}), 6.93-6.98(\mathrm{~m}, 2 \mathrm{H}, \mathrm{ArH}), 7.27-7.31\left(\mathrm{~m}, 2 \mathrm{H}, \mathrm{C}_{6} \mathrm{H}_{5}\right), 7.40(\mathrm{tt}, 1 \mathrm{H}, \mathrm{J} 7.4,1.2$ $\left.\mathrm{Hz}, \mathrm{C}_{6} \mathrm{H}_{5}\right), 7.47-7.52\left(\mathrm{~m}, 2 \mathrm{H}, \mathrm{C}_{6} \mathrm{H}_{5}\right) \mathrm{ppm} .{ }^{13} \mathrm{C} \mathrm{NMR}\left(100 \mathrm{MHz}, \mathrm{CDCl}_{3}, 23{ }^{\circ} \mathrm{C}\right): \delta \mathrm{ppm}=14.87\left(\mathrm{CH}_{3}\right), 19.87\left(\mathrm{CH}_{2}-3\right)$, 39.47, $39.92\left(\mathrm{CH}_{2}-2, \mathrm{CH}_{2}-4\right), 55.60\left(\mathrm{CH}_{2}-10\right), 61.91(\mathrm{C}-5), 63.78\left(\mathrm{OCH}_{2}\right), 72.08\left(\mathrm{CH}_{2}-8\right), 115.48,119.97(\mathrm{ArH})$, 126.82, 128.52, $130.03\left(\mathrm{C}_{6} \mathrm{H}_{5}\right), 141.37,143.75\left(\mathrm{C}_{6} \mathrm{H}_{5}, \mathrm{Ar}\right), 154.70(\mathrm{Ar}), 202.88(\mathrm{C}=\mathrm{S}), 215.68(\mathrm{C}=0)$ ppm. Anal. Calcd for $\mathrm{C}_{22} \mathrm{H}_{24} \mathrm{~N}_{2} \mathrm{O}_{2} \mathrm{~S}$ : C, 69.44; $\mathrm{H}, 6.36 ; \mathrm{N}, 7.36 ; \mathrm{S}, 8.43$. Found: $\mathrm{C}, 69.24 ; \mathrm{H}, 6.53 ; \mathrm{N}, 7.44 ; \mathrm{S}, 8.39 \%$.

4-Benzyl-2-phenyl-1-sulfanylidene-2,4-diazaspiro[5.5] undecan-7-one (4d). Pale yellow crystals; yield: 82\% (DMSO); mp 132-134 ${ }^{\circ} \mathrm{C}$ (from EtOH). IR (ATR, $\mathrm{v}_{\max } \mathrm{cm}^{-1}$ ): 3063, 3026, 2925 (CH); 1710 (CO). ${ }^{1} \mathrm{H} \mathrm{NMR} \mathrm{(400} \mathrm{MHz,}$ $\left.\mathrm{CDCl}_{3}, 23^{\circ} \mathrm{C}\right): \delta=1.39-1.54(\mathrm{~m}, 1 \mathrm{H}, \mathrm{C} \underline{\mathrm{H}}-10), 1.81-1.91\left(\mathrm{~m}, 3 \mathrm{H}, \mathrm{CH} \underline{\mathrm{H}}-10, \mathrm{CH}_{2}-9\right), 2.05(\mathrm{dm}, 1 \mathrm{H}, J \mathrm{ca} .14 .0 \mathrm{~Hz}$, CㅂH-11), 2.21 (ddd, $1 \mathrm{H}, J$ 15.6, 9.3, 8.1 Hz, CㅂH-8), 2.57 (dtd, $1 \mathrm{H}, J$ J 15.6, 4.6, $1.5 \mathrm{~Hz}, \mathrm{CH} \underline{\mathrm{H}}-8$ ), 2.83 (d, $1 \mathrm{H}, J 12.5$ $\mathrm{Hz}, \underline{\mathrm{C}} \underline{\mathrm{H}}-5$ ), 3.14 (ddd, $1 \mathrm{H}, J$ 14.0, 13.2, $4.0 \mathrm{~Hz}, \mathrm{CH} \underline{\mathrm{H}}-11$ ), 3.38 (d, $1 \mathrm{H}, J 12.5 \mathrm{~Hz}, \mathrm{CH} \underline{\mathrm{H}}-5), 3.66$ (d, $1 \mathrm{H}, J 13.2 \mathrm{~Hz}$, $\mathrm{NC} \underline{\mathrm{H}}$ ), 3.76 (d, $1 \mathrm{H}, J 13.2 \mathrm{~Hz}, \mathrm{NCH} \underline{\mathrm{H}}), 4.25$ (dd, $1 \mathrm{H}, J$ 10.5, $0.9 \mathrm{~Hz}, \mathrm{C} \underline{H} H-3), 4.37$ (dd, $1 \mathrm{H}, J 10.5,0.6 \mathrm{~Hz}, \mathrm{CH} \underline{\mathrm{H}}-3$ ), 7.25 - $7.38\left(\mathrm{~m}, 8 \mathrm{H}, 2 \times \mathrm{C}_{6} \mathrm{H}_{5}\right), 7.43-7.49\left(\mathrm{~m}, 2 \mathrm{H}, \mathrm{C}_{6} \mathrm{H}_{5}\right) \mathrm{ppm} .{ }^{13} \mathrm{C} \mathrm{NMR}\left(100 \mathrm{MHz}, \mathrm{CDCl}_{3}, 23{ }^{\circ} \mathrm{C}\right): \delta \mathrm{ppm}=20.52$ ( $\left.\mathrm{CH}_{2}-10\right), 24.92\left(\mathrm{CH}_{2}-9\right), 37.80\left(\mathrm{CH}_{2}-11\right), 38.81\left(\mathrm{CH}_{2}-8\right), 55.95\left(\mathrm{CH}_{2}-5\right), 58.36\left(\mathrm{NCH}_{2}\right), 62.06(\mathrm{C}-6), 74.57\left(\mathrm{CH}_{2}-3\right)$, 126.94, 127.90, 128.22, 128.65, 128.79, 129.88, 136.32, $145.00\left(2 \times \mathrm{C}_{6} \mathrm{H}_{5}\right), 202.98$ ( $\left.\mathrm{C}=\mathrm{S}\right), 208.67$ (C=O) ppm. Anal. Calcd for $\mathrm{C}_{22} \mathrm{H}_{24} \mathrm{~N}_{2} \mathrm{OS}: \mathrm{C}, 72.49 ; \mathrm{H}, 6.64 ; \mathrm{N}, 7.69 ; \mathrm{S}, 8.80$. Found: $\mathrm{C}, 72.20 ; \mathrm{H}, 6.98 ; \mathrm{N}, 7.69 ; \mathrm{S}, 9.08 \%$. 
2-(4-Chlorophenyl)-4-(2-phenylethyl)-1-sulfanylidene-2,4-diazaspiro[5.5]undecan-7-one (4e). Yellowish semisolid; yield: 61\%. IR (ATR, $\left.v_{\max } \mathrm{Cm}^{-1}\right): 3059,3026,2930,2863(\mathrm{CH}) ; 1707(\mathrm{CO}) .{ }^{1} \mathrm{H} \mathrm{NMR}\left(400 \mathrm{MHz}, \mathrm{CDCl}_{3}, 23^{\circ} \mathrm{C}\right): \delta$ $=1.57-1.70(\mathrm{~m}, 1 \mathrm{H}, \mathrm{C} \underline{\mathrm{H}} \mathrm{H}-10), 1.88-2.08$ (m, $\left.4 \mathrm{H}, \mathrm{CH}_{2}-9, \mathrm{CH} \underline{\mathrm{H}}-10, \mathrm{C} \underline{\mathrm{H}}-11\right), 2.43$ (ddd, $1 \mathrm{H}, J 15.6,10.9,6.5 \mathrm{~Hz}$,

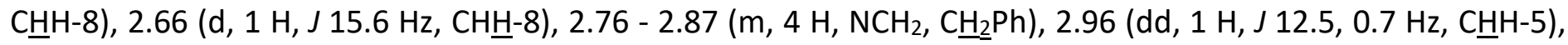
3.08 (ddd, $1 \mathrm{H}, J$ 13.7, 13.1, 3.9 Hz, CHㅡ-5), 3.46 (d, $1 \mathrm{H}, J 12.5 \mathrm{~Hz}, \mathrm{CH} \underline{\mathrm{H}}-5), 4.22$ (dd, $1 \mathrm{H}, \mathrm{J} 10.5,1.0 \mathrm{~Hz}, \mathrm{C} \underline{\mathrm{H}}-3$ ), $4.33(\mathrm{~d}, 1 \mathrm{H}, \mathrm{J} 10.5 \mathrm{~Hz}, \mathrm{CH} \underline{\mathrm{H}}-3), 7.16-7.25\left(\mathrm{~m}, 5 \mathrm{H}, \mathrm{ArH}, \mathrm{C}_{6} \mathrm{H}_{5}\right), 7.26-7.33\left(\mathrm{~m}, 2 \mathrm{H}, \mathrm{C}_{6} \mathrm{H}_{5}\right), 7.39-7.46(\mathrm{~m}, 2 \mathrm{H}, \mathrm{ArH})$ ppm. ${ }^{13} \mathrm{C}$ NMR $\left(100 \mathrm{MHz}, \mathrm{CDCl}_{3}, 23^{\circ} \mathrm{C}\right): \delta \mathrm{ppm}=20.64\left(\mathrm{CH}_{2}-10\right), 24.89\left(\mathrm{CH}_{2}-9\right), 33.85\left(\mathrm{CH}_{2} \mathrm{Ph}\right), 37.98\left(\mathrm{CH}_{2}-11\right)$, $39.01\left(\mathrm{CH}_{2}-8\right), 55.85\left(\mathrm{NCH}_{2}\right), 56.84\left(\mathrm{CH}_{2}-5\right), 61.95$ (C-6), $74.27\left(\mathrm{CH}_{2}-3\right), 126.51,128.42,128.59,128.61,130.20$, 134.01, 138.97, $143.41\left(\mathrm{C}_{6} \mathrm{H}_{5}, \mathrm{ArH}\right), 203.36$ (C=S), 208.85 (C=O) ppm. Anal. Calcd for $\mathrm{C}_{23} \mathrm{H}_{25} \mathrm{ClN}_{2} \mathrm{OS}: \mathrm{C}, 66.89 ; \mathrm{H}$, $6.10 ; \mathrm{N}, 6.78 ; \mathrm{S}, 7.76$. Found: $\mathrm{C}, 66.66 ; \mathrm{H}, 6.05 ; \mathrm{N}, 6.42 ; \mathrm{S}, 7.53 \%$.

2-(4-Chlorophenyl)-4-(4-methylphenyl)-1-sulfanylidene-2,4-diazaspiro[5.5]undecan-7-one (4f). Pale yellow crystals; yield: $72 \%$; $m p ~ 116-118{ }^{\circ} \mathrm{C}$ (from EtOH). IR (ATR, $v_{\max } \mathrm{cm}^{-1}$ ): 2943, 2922, 2856 (CH), 1704 (CO). ${ }^{1} \mathrm{H}$ NMR $\left(400 \mathrm{MHz}, \mathrm{CDCl}_{3}, 23{ }^{\circ} \mathrm{C}\right): \delta=1.68-1.81(\mathrm{~d}, 1 \mathrm{H}, J 3.9 \mathrm{~Hz}, \underline{\mathrm{C}} \underline{\mathrm{H}}-10), 1.86-2.10\left(\mathrm{~m}, 4 \mathrm{H}, \mathrm{CH} \underline{\mathrm{H}}-10, \mathrm{C}_{\mathbf{H}} \mathrm{H}-11, \mathrm{CH}_{2}-9\right)$, $2.28\left(\mathrm{~s}, 3 \mathrm{H}, \mathrm{CH}_{3}\right), 2.51$ (ddd, $\left.1 \mathrm{H}, \mathrm{J} 15.8,11.0,5.7 \mathrm{~Hz}, \mathrm{C} \underline{\mathrm{H}} \mathrm{H}-8\right), 2.66$ (dt, $1 \mathrm{H}, J$ 15.8, 5.0 Hz, CHㅡ-8), 2.96 - 3.08 (m, $1 \mathrm{H}, \mathrm{CH} \underline{\mathrm{H}}-11$ ), 3.59 (d, $1 \mathrm{H}, J 12.5 \mathrm{~Hz}, \mathrm{C} \underline{\mathrm{H}}-5), 4.16$ (d, $1 \mathrm{H}, J 12.6 \mathrm{~Hz}, \mathrm{CH} \underline{\mathrm{H}}-5), 4.82$ (d, $1 \mathrm{H}, J 11.1 \mathrm{~Hz}, \mathrm{CHH}-3), 4.91$ (d, $1 \mathrm{H}, J 11.1 \mathrm{~Hz}, \mathrm{CH} \underline{\mathrm{H}}-3), 6.74$ (d, $2 \mathrm{H}, J 8.4 \mathrm{~Hz}, \mathrm{ArH}), 7.10$ (d, $2 \mathrm{H}, J 8.4 \mathrm{~Hz}, \mathrm{ArH}), 7.23-7.28(\mathrm{~m}, 2 \mathrm{H}, \mathrm{ArH}), 7.42-$ $7.48(\mathrm{~m}, 2 \mathrm{H}, \mathrm{ArH}) .{ }^{13} \mathrm{C} \mathrm{NMR}\left(100 \mathrm{MHz}, \mathrm{CDCl}_{3}, 23^{\circ} \mathrm{C}\right): \delta=20.47\left(\mathrm{CH}_{3}\right), 20.84\left(\mathrm{CH}_{2}-10\right), 25.48\left(\mathrm{CH}_{2}-9\right), 37.16\left(\mathrm{CH}_{2^{-}}\right.$ 11), $39.22\left(\mathrm{CH}_{2}-8\right), 53.53\left(\mathrm{CH}_{2}-5\right), 62.47(\mathrm{C}-6), 69.96\left(\mathrm{CH}_{2}-3\right), 115.89,128.45,130.12,130.30(\operatorname{ArH}), 130.73$, 134.23, 143.24, 144.99 (Ar), 204.00 (C=S), 208.59 (C=O). Anal. Calcd for $\mathrm{C}_{22} \mathrm{H}_{23} \mathrm{ClN}_{2} \mathrm{OS}: \mathrm{C}, 66.23 ; \mathrm{H}, 5.81 ; \mathrm{N}, 7.02$; S, 8.04. Found: C, 66.57; H, 6.27; N, 6.99; S, 8.38\%.

4-[(4-Methylphenyl)methyl]-2-phenyl-1-sulfanylidene-2,4-diazaspiro[5,6]dodecan-7-one (4g). White crystals; yield: 94\%; mp 114-116 ${ }^{\circ} \mathrm{C}$ (from EtOH). IR (ATR, $\mathrm{V}_{\max } \mathrm{Cm}^{-1}$ ): 2973, 2923, 2855 (CH), 1686 (CO). ${ }^{1} \mathrm{H} \mathrm{NMR} \mathrm{(400} \mathrm{MHz,}$ $\mathrm{CDCl}_{3}, 2{ }^{\circ} \mathrm{C}$ ): $\delta=1.12$ (tdt, J ca. 13.9, ca. 12.1, ca. $\left.2.2 \mathrm{~Hz}, 1 \mathrm{H}, \mathrm{C} \underline{\mathrm{H}}-9\right), 1.19-1.29(\mathrm{~m}, 1 \mathrm{H}, \mathrm{C} \underline{\mathrm{H}} \mathrm{H}-11), 1.51$ (dtt, J ca. 13.4, ca. 12.2, ca. $3.2 \mathrm{~Hz}, 1 \mathrm{H}, \mathrm{C} \underline{\mathrm{H}} \mathrm{H}-10), 1.77$ (dquin, J 13.4, ca. $3.3 \mathrm{~Hz}, 1 \mathrm{H}, \mathrm{CH} \underline{\mathrm{H}}-10), 1.82$ - 1.95 (m, $2 \mathrm{H}, \mathrm{CH} \underline{\mathrm{H}}-$ 9, $\mathrm{CH} \underline{\mathrm{H}}-11$ ), 2.34 (s, $3 \mathrm{H}, \mathrm{CH}_{3}$ ), 2.40 (dd, J 14.5, $\left.7.9 \mathrm{~Hz}, 1 \mathrm{H}, \underline{\mathrm{CH}} \mathrm{H}-8\right), 2.59$ (ddt, J 12.3, 7.4, ca. 0.7 Hz, $1 \mathrm{H}, \mathrm{C} \underline{\mathrm{H}} \mathrm{H}-12$ ), 2.73 (ddt, J 14.5, 11.6, ca. $0.7 \mathrm{~Hz}, 1 \mathrm{H}, \mathrm{CH} \underline{\mathrm{H}}-8$ ), 2.99 (dd, J 12.7, $1.3 \mathrm{~Hz}, 1 \mathrm{H}, \mathrm{C} \underline{\mathrm{H}} \mathrm{H}-5), 3.40$ (d, J $12.7 \mathrm{~Hz}, 1 \mathrm{H}, \mathrm{CH} \underline{\mathrm{H}}-$ 5), 3.46 (td, J 12.3, $2.2 \mathrm{~Hz}, 1 \mathrm{H}, \mathrm{CH} \underline{\mathrm{H}}-12), 3.66$ (d, J $13.2 \mathrm{~Hz}, 1 \mathrm{H}, \mathrm{NC} \underline{H} \mathrm{H}), 3.77$ (d, J $13.2 \mathrm{~Hz}, 1 \mathrm{H}, \mathrm{NCH} \underline{\mathrm{H}}), 4.28$ (d, J $10.6 \mathrm{~Hz}, 1 \mathrm{H}, \mathrm{C} \underline{\mathrm{H}}-3), 4.35$ (dd, J 10.6, $1.3 \mathrm{~Hz}, 1 \mathrm{H}, \mathrm{CH} \underline{\mathrm{H}}-3), 7.12-7.19(\mathrm{~m}, 4 \mathrm{H}, \mathrm{ArH}), 7.22-7.27(\mathrm{~m}, 2 \mathrm{H}, \mathrm{ArH})$, 7.34 (tt, J 7.3, $1.5 \mathrm{~Hz}, 1 \mathrm{H}, \mathrm{ArH}), 7.41-7.46(\mathrm{~m}, 2 \mathrm{H}, \mathrm{ArH}) \mathrm{ppm} ;{ }^{13} \mathrm{C} \mathrm{NMR}\left(100 \mathrm{MHz}, \mathrm{CDCl}_{3}, 23{ }^{\circ} \mathrm{C}\right): \delta \mathrm{ppm}=21.14$ $\left(\mathrm{CH}_{3}\right), 25.16\left(\mathrm{CH}_{2}-9\right), 26.17\left(\mathrm{CH}_{2}-11\right), 30.41\left(\mathrm{CH}_{2}-10\right), 37.66\left(\mathrm{CH}_{2}-8\right), 45.11\left(\mathrm{CH}_{2}-12\right), 53.97\left(\mathrm{CH}_{2}-5\right), 58.00\left(\mathrm{NCH}_{2}\right)$, 63.44 (C-6), 74.75 ( $\left.\mathrm{CH}_{2}-3\right), 126.82,128.22,128.70,129.22,129.87,133.60,137.37,144.47\left(\mathrm{C}_{6} \mathrm{H}_{5}, \mathrm{C}_{6} \mathrm{H}_{4}\right), 203.20$ (C=S), 212.23 (C=O) ppm. Anal. Calcd for $\mathrm{C}_{24} \mathrm{H}_{28} \mathrm{~N}_{2} \mathrm{OS}$ : C, 73.43; $\mathrm{H}, 7.19 ; \mathrm{N}, 7.14 ; \mathrm{S}, 8.17$. Found: C, 73.36; $\mathrm{H}, 7.58$; $\mathrm{N}, 7.13 ; \mathrm{S}, 8.31 \%$.

4-(4-Methoxybenzyl)-1-sulfanylidene-2-p-tolyl-2,4-diazaspiro[5.7]tridecan-7-one (4h). Yellow crystals; yield: 95\% (DMSO), 84\% (EtOH); mp 108-110 ${ }^{\circ} \mathrm{C}$ (from EtOH). IR (ATR, $v_{\max } \mathrm{cm}^{-1}$ ): 2992, 2979, 2919 (CH), 1690 (CO). ${ }^{1} \mathrm{H}$ NMR (400 MHz, CDCl $\left.3,23{ }^{\circ} \mathrm{C}\right): \delta=0.74-0.86(\mathrm{~m}, 1 \mathrm{H}, \underline{\mathrm{C}} \underline{\mathrm{H}}-11), 1.29-1.74\left(\mathrm{~m}, 6 \mathrm{H}, \mathrm{CH}_{2}-9, \mathrm{CH}_{2}-10, \mathrm{CH} \underline{\mathrm{H}}-11, \mathrm{C} \underline{\mathrm{H}} \mathrm{H}-\right.$ 12), 1.82-192 (m, $1 \mathrm{H}, \mathrm{CH} \underline{\mathrm{H}}-12$ ), 2.23 (dt, J 14.9, 3.9 Hz, $1 \mathrm{H}, \mathrm{C} \underline{\mathrm{H}} \mathrm{H}-8$ ), 2.35 (s, $3 \mathrm{H}, \mathrm{CH}_{3}$ ), 2.38 (ddd, J 12.5, 5.6, 2.7 $\mathrm{Hz}, 1 \mathrm{H}, \underline{\mathrm{C}} \underline{\mathrm{H}}-13$ ), 2.92 (d, J $12.7 \mathrm{~Hz}, 1 \mathrm{H}, \mathrm{C} \underline{\mathrm{H}}-5$ ), 3.13 (ddd, J 14.9, 13.2, 4.2 Hz, $1 \mathrm{H}, \mathrm{CH} \underline{\mathrm{H}}-8$ ), 3.64 (d, J $12.7 \mathrm{~Hz}, 1$ $\mathrm{H}, \mathrm{CH} \underline{\mathrm{H}}-5), 3.66$ - $3.74\left(\mathrm{~m}, \mathrm{NCH}_{2}, 3 \mathrm{H}, \mathrm{CH} \underline{\mathrm{H}}-13\right), 3.80\left(\mathrm{~s}, 3 \mathrm{H}, \mathrm{OCH}_{3}\right), 4.20-4.28\left(\mathrm{~m}, 2 \mathrm{H}, \mathrm{CH}_{2}-3\right), 6.86(\mathrm{~d}, J 8.5 \mathrm{~Hz}, 2$ $\mathrm{H}, \mathrm{ArH}), 6.99(\mathrm{~d}, J 8.1 \mathrm{~Hz}, 2 \mathrm{H}, \mathrm{ArH}), 7.20-7.28(\mathrm{~m}, 4 \mathrm{H}, \mathrm{ArH}) \mathrm{ppm} ;{ }^{13} \mathrm{C} \mathrm{NMR}\left(100 \mathrm{MHz}, \mathrm{CDCl}_{3}, 23{ }^{\circ} \mathrm{C}\right): \delta \mathrm{ppm}=$ $21.23\left(\mathrm{CH}_{3}\right), 24.74\left(\mathrm{CH}_{2}-11, \mathrm{CH}_{2}-9\right), 26.75\left(\mathrm{CH}_{2}-10\right), 30.56\left(\mathrm{CH}_{2}-12\right), 35.57\left(\mathrm{CH}_{2}-8\right), 40.82\left(\mathrm{CH}_{2}-13\right), 51.68\left(\mathrm{CH}_{2}-5\right)$, $55.28\left(\mathrm{OCH}_{3}\right), 58.51\left(\mathrm{NCH}_{2}\right), 62.46(\mathrm{C}-6), 74.60\left(\mathrm{CH}_{2}-3\right), 113.86,126.33,128.77,130.18,130.55,138.08,142.20$, $159.14\left(2 \times \mathrm{C}_{6} \mathrm{H}_{4}\right), 201.79(\mathrm{C}=\mathrm{S}), 215.00(\mathrm{C}=\mathrm{O}) \mathrm{ppm}$. Anal. Calcd for $\mathrm{C}_{26} \mathrm{H}_{32} \mathrm{~N}_{2} \mathrm{O}_{2} \mathrm{~S}: \mathrm{C}, 71.52 ; \mathrm{H}, 7.39 ; \mathrm{N}, 6.42 ; \mathrm{S}$, 7.34. Found: $C, 71.82 ; H, 7.62 ; N, 6.43 \mathrm{~S}, 7.43 \%$. 
4-Benzyl-1-thioxo-2-p-tolyl-2,4-diazaspiro[5.7]tridecan-7-one (4i). White crystals; yield: $87 \%$; $\mathrm{mp} 124-126{ }^{\circ} \mathrm{C}$ (from EtOH). IR (ATR, $\left.v_{\max } \mathrm{cm}^{-1}\right):$ 2919, 2856,2823 (CH), $1687(\mathrm{CO}) .{ }^{1} \mathrm{H}$ NMR (400 MHz): $\delta=0.73-0.86(\mathrm{~m}, 1 \mathrm{H}$, CㅂH-11), 1.27-1.74 (m, $\left.6 \mathrm{H}, \mathrm{CH}_{2}-9, \mathrm{CH}_{2}-10, \mathrm{C} \underline{\mathrm{H}} \mathrm{H}-11, \mathrm{C} \underline{\mathrm{H}} \mathrm{H}-12,\right), 1.82$ - 1.91 (m, $\left.1 \mathrm{H}, \mathrm{C} \underline{\mathrm{H}} \mathrm{H}-12\right), 2.24$ (dt, $1 \mathrm{H}, \mathrm{J}$ 15.0, $4.0 \mathrm{~Hz}, \mathrm{C} \underline{\mathrm{H}} \mathrm{H}-8$ ), 2.34 (s, $3 \mathrm{H}, \mathrm{CH}_{3}$ ), 2.38 (ddd, $\left.1 \mathrm{H}, \mathrm{J} 12.5,5.9,3.1 \mathrm{~Hz}, \mathrm{CH} \underline{\mathrm{H}}-8\right), 2.93$ (dd, $\left.1 \mathrm{H}, J 12.7,0.9 \mathrm{~Hz}, \mathrm{CH} \underline{\mathrm{H}}-5\right)$, 3.14 (ddd, $1 \mathrm{H}, J$ 15.0, 13.3, $4.4 \mathrm{~Hz}, \mathrm{CH} \underline{\mathrm{H}}-8), 3.65$ - $3.70(\mathrm{~m}, 1 \mathrm{H}, \mathrm{J} 12.6 \mathrm{~Hz}, \mathrm{C} \underline{\mathrm{H}} \mathrm{H}-5), 3.65-3.80(\mathrm{~m}, 3 \mathrm{H}, \mathrm{NCH}, \mathrm{C} \underline{\mathrm{H}}-$ 13), 4.22 - $4.30\left(\mathrm{~m}, 2 \mathrm{H}, \mathrm{CH}_{2}-3\right), 6.97-7.01(\mathrm{~m}, 2 \mathrm{H}, \mathrm{ArH}), 7.20-7.38(\mathrm{~m}, 7 \mathrm{H}, \mathrm{ArH}) ;{ }^{13} \mathrm{C} \mathrm{NMR}\left(100 \mathrm{MHz}, \mathrm{CDCl}_{3}, 23\right.$ $\left.{ }^{\circ} \mathrm{C}\right): \delta \mathrm{ppm}=21.22\left(\mathrm{CH}_{3}\right), 24.73\left(\mathrm{CH}_{2}-11, \mathrm{CH}_{2}-9\right), 26.74\left(\mathrm{CH}_{2}-10\right), 30.53\left(\mathrm{CH}_{2}-12\right), 35.60\left(\mathrm{CH}_{2}-8\right), 40.78\left(\mathrm{CH}_{2}-13\right)$, $51.87\left(\mathrm{CH}_{2}-5\right), 59.14\left(\mathrm{NCH}_{2}\right), 62.49(\mathrm{C}-6), 74.62\left(\mathrm{CH}_{2}-3\right), 126.33,127.72,128.52,128.98,130.55(\operatorname{ArH}), 136.82$, 138.08, 142.18 ( $\mathrm{Ar}), 201.77(\mathrm{C}=\mathrm{S}), 214.91(\mathrm{C}=\mathrm{O})$. Anal. Calcd for $\mathrm{C}_{25} \mathrm{H}_{30} \mathrm{~N}_{2} \mathrm{OS}$ : C, 73.85; H, 7.44; N, 6.89; $\mathrm{S}, 7.89$. Found: $\mathrm{C}, 73.88 ; \mathrm{H}, 7.79 ; \mathrm{N}, 6.93 ; \mathrm{S}, 7.96 \%$.

4-Benzyl-2-phenyl-1-sulfanylidene-2,4-diazaspiro[5.11] hepta-decan-7-one (4j). Pale yellow crystals; yield: 86

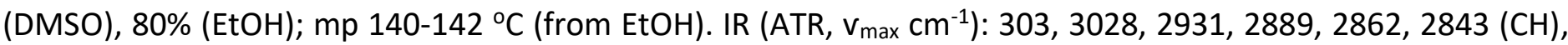
1708 (CO). ${ }^{1} \mathrm{H}$ NMR $\left(400 \mathrm{MHz}, \mathrm{CDCl}_{3}, 23{ }^{\circ} \mathrm{C}\right): \delta=0.91-1.04(\mathrm{~m}, 1 \mathrm{H}, \mathrm{C} \underline{\mathrm{HH}}-16), 1.08-1.41(\mathrm{~m}, 14 \mathrm{H}, \underline{\mathrm{C}} \underline{\mathrm{H}}-\mathbf{9}, \mathrm{CH} \underline{\mathrm{H}}-$ 16, $\left.\mathrm{CH}_{2}-10, \mathrm{CH}_{2}-11, \mathrm{CH}_{2}-12, \mathrm{CH}_{2}-13, \mathrm{CH}_{2}-14, \mathrm{CH}_{2}-15\right), 1.77$ (ddd, J 14.6, 13.2, $6.6 \mathrm{~Hz}, 1 \mathrm{H}, \mathrm{C} \underline{\mathrm{H}}-17$ ), 2.10 - 2.19 (m, $1 \mathrm{H}, \mathrm{CH} \underline{\mathrm{H}}-9$ ), 2.28 (dt, J 19.0, $2.9 \mathrm{~Hz}, 1 \mathrm{H}, \underline{\mathrm{CH}}-8$ ), 2.44 (d, J $12.0 \mathrm{~Hz}, 1 \mathrm{H}, \mathrm{C} \underline{\mathrm{H}}-5), 2.78$ (ddd, J 15.1, 13.2, $2.0 \mathrm{~Hz}, 1$ $\mathrm{H}, \mathrm{CH} \underline{\mathrm{H}}-17$ ), 3.67 (ddd, J 19.0, 12.5, $2.2 \mathrm{~Hz}, 1 \mathrm{H}, \mathrm{CH} \underline{\mathrm{H}}-8), 3.83$ (d, J $13.4 \mathrm{~Hz}, 1 \mathrm{H}, \mathrm{NC} \underline{\mathrm{H}} \mathrm{H}), 3.87$ (d, J $13.4 \mathrm{~Hz}, 1 \mathrm{H}$, $\mathrm{NCH} \underline{\mathrm{H}}), 4.10$ (d, J $12.0 \mathrm{~Hz}, 1 \mathrm{H}, \mathrm{CH} \underline{\mathrm{H}}-5), 4.13$ (dd, J 11.7, 0.7 Hz, $1 \mathrm{H}, \underline{\mathrm{C}} \underline{\mathrm{H}}-3), 4.33$ (dd, J 11.7, 0.7 Hz, $1 \mathrm{H}, \mathrm{CH} \underline{\mathrm{H}}-3)$, 7.14 - $7.18(\mathrm{~m}, 2 \mathrm{H}, \mathrm{ArH}), 7.24-7.36(\mathrm{~m}, 6 \mathrm{H}, \mathrm{ArH}), 7.41-7.47(\mathrm{~m}, 2 \mathrm{H}, \mathrm{ArH}) \mathrm{ppm} .{ }^{13} \mathrm{C} \mathrm{NMR}\left(100 \mathrm{MHz}, \mathrm{CDCl}_{3}, 23\right.$ $\left.{ }^{\circ} \mathrm{C}\right): \delta \mathrm{ppm}=19.51\left(\mathrm{CH}_{2}-16\right), 21.82,21.97,22.16,22.61,23.48,26.49,26.61\left(7 \times \mathrm{CH}_{2}\right), 34.24\left(\mathrm{CH}_{2}-8\right), 37.22\left(\mathrm{CH}_{2}-\right.$ 17), $53.11\left(\mathrm{CH}_{2}-5\right), 60.08\left(\mathrm{NCH}_{2}\right), 64.73(\mathrm{C}-6), 72.18\left(\mathrm{CH}_{2}-3\right), 126.19,127.57,128.05,128.51,128.70,129.86$, 137.04, $145.58\left(2 \times \mathrm{C}_{6} \mathrm{H}_{5}\right), 203.18$ (C=S), 206.29 (C=O) ppm. Anal. Calcd for $\mathrm{C}_{28} \mathrm{H}_{36} \mathrm{~N}_{2} \mathrm{OS}$ : C, 74.96; $\mathrm{H}, 8.09 ; \mathrm{N}, 6.24$; $S, 7.15$. Found: $C, 74.58 ; H, 8.48 ; N, 6.13 ; S, 7.11 \%$.

4-(Methylpropyl)-2-(naphthalen-1-yl)-1-sulfanylidene-2,4-diazaspiro[5.11]heptadecan-7-one (4k). $\quad 1: 1$ mixture of rotamers: Pale yellow crystals; yield: $85 \%$; $\mathrm{mp} 80-82{ }^{\circ} \mathrm{C}$ (from aq EtOH). IR (ATR, $v_{\max } \mathrm{cm}^{-1}$ ): 3505 , 2928, 2865 (CH), 1707 (CO). ${ }^{1} \mathrm{H}$ NMR (400 MHz, CDCl $\left.3,23{ }^{\circ} \mathrm{C}\right): \delta=0.88-0.97\left(\mathrm{~m}, 12 \mathrm{H}, 2 \times \mathrm{CH}\left(\mathrm{C}_{3}\right)_{2}\right), 1.01-1.46$ ( $\mathrm{m}, 30 \mathrm{H}, 14 \times \mathrm{CH}_{2}, 2 \times \mathrm{C} \underline{\mathrm{H}}$ ), $1.71-1.77$ ( $\left.\mathrm{m}, 1 \mathrm{H}, \mathrm{C} \underline{\mathrm{H}}-17\right), 1.80$ (spt, J 6.7 Hz, $\left.2 \mathrm{H}, 2 \times \mathrm{C} \underline{\mathrm{H}}\left(\mathrm{CH}_{3}\right)_{2}\right), 1.93$ (ddd, J 14.7, 13.2, $6.6 \mathrm{~Hz}, 1 \mathrm{H}, \mathrm{C} \underline{\mathrm{H}}-17), 2.11-2.22(\mathrm{~m}, 2 \mathrm{H}, 2 \times \mathrm{CH} \underline{\mathrm{H}}), 2.25-2.48\left(\mathrm{~m}, 7 \mathrm{H}, 2 \times \mathrm{NCH}_{2}, \underline{\mathrm{C}} \underline{\mathrm{H}}-5, \mathrm{C} \underline{\mathrm{H}} \mathrm{H}-8, \mathrm{C} \underline{\mathrm{H}} \mathrm{H}-8\right)$, $2.72-2.82(\mathrm{~m}, 2 \mathrm{H}, \mathrm{C} \underline{\mathrm{H}} \mathrm{H}-5, \mathrm{CH} \underline{\mathrm{H}}-17), 2.93$ (ddd, J 15.1, 12.9, $2.2 \mathrm{~Hz}, 1 \mathrm{H}, \mathrm{CH} \underline{\mathrm{H}}-17$ ), 3.71 (ddd, J 18.8, 12.0, 2.2 Hz,

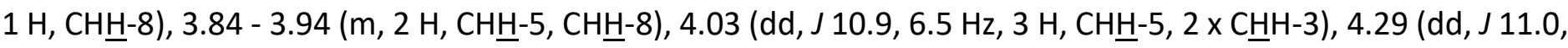
$1.1 \mathrm{~Hz}, 1 \mathrm{H}, \mathrm{CH} \underline{\mathrm{H}}-3$ ), 4.37 (dd, J 10.9, $1.1 \mathrm{~Hz}, 1 \mathrm{H}, \mathrm{CH} \underline{\mathrm{H}}-3$ ), 7.23 (dd, J 7.1, $1.0 \mathrm{~Hz}, 1 \mathrm{H}, \mathrm{ArH}), 7.36$ (dd, J 7.3, $1.0 \mathrm{~Hz}$, $1 \mathrm{H}, \mathrm{ArH}), 7.47-7.57(\mathrm{~m}, 7 \mathrm{H}, \mathrm{ArH}), 7.85-8.00(\mathrm{~m}, 5 \mathrm{H}, \mathrm{ArH}) \mathrm{ppm} .{ }^{13} \mathrm{C} \mathrm{NMR}\left(100 \mathrm{MHz}, \mathrm{CDCl}_{3}, 23{ }^{\circ} \mathrm{C}\right): \delta \mathrm{ppm}=$ 19.66 and $19.93\left(2 \times \mathrm{CH}_{2}\right), 20.43$ and $20.53\left(2 \times \mathrm{CH}_{3}\right), 20.75\left(2 \times \mathrm{CH}_{3}\right), 21.88$ and $22.04\left(2 \times \mathrm{CH}_{2}\right), 22.21$ and 22.33 ( $\left.2 \times \mathrm{CH}_{2}\right), 22.64$ and $22.74\left(2 \times \mathrm{CH}_{2}\right), 23.54$ and $23.66\left(2 \times \mathrm{CH}_{2}\right), 26.05$ and 26.33 [ $\left.2 \times \mathrm{C} \mathrm{H}\left(\mathrm{CH}_{3}\right) 2\right], 26.52$ and 26.63 ( $2 \times \mathrm{CH}_{2}$ ), 34.31 and $35.20\left(2 \times \mathrm{CH}_{2}-8\right), 37.08$ and $37.82\left(2 \times \mathrm{CH}_{2}-17\right), 53.03$ and $54.53\left(2 \times \mathrm{CH}_{2}-5\right), 63.47$ and 63.67 $\left(\mathrm{NCH}_{2}\right), 64.71$ and 6512 (2x C-6), 72.06 and $74.30\left(2 \times \mathrm{CH}_{2}-3\right), 121.36,123.08,124.14,124.66,125.68,125.76$, $126.61,126.67,127.12,127.40,127.94,128.48,128.58,128.75,128.96,134.76,134.85,141.77,142.08(2 \times$ ArH), 203.54 and 203.60 ( $2 \times \mathrm{C}=\mathrm{S}$ ), 206.29 and 206.52 (C=O). Anal. Calcd for $\mathrm{C}_{28} \mathrm{H}_{38} \mathrm{~N}_{2} \mathrm{OS}: \mathrm{C}, 74.62 ; \mathrm{H}, 8.50 ; \mathrm{N}$, 6.22; S, 7.11. Found: C, 74.57; H, 8.91; N, 6.34; S, 7.34\%.

4-(4-Ethoxyphenyl)-2-phenyl-1-sulfanylidene-2,4-diazaspiro[5.11]heptadecan-7-one (4I). Pale yellow crystals; yield: 62\%; mp 87-90 ${ }^{\circ} \mathrm{C}$ (from EtOH). IR (ATR, $\mathrm{V}_{\max } \mathrm{cm}^{-1}$ ): 2928, $2863(\mathrm{CH}), 1707$ (CO). ${ }^{1} \mathrm{H} \mathrm{NMR}\left(400 \mathrm{MHz} \mathrm{CDCl}_{3}\right.$, $\left.23^{\circ} \mathrm{C}\right): \delta=1.02-1.45\left(\mathrm{~m}, 18 \mathrm{H}, \mathrm{CH}_{3}, 14 \times \mathrm{CH}_{2}, \underline{\mathrm{CH}}-9\right)$ ), 1.86 (ddd, $1 \mathrm{H}, J 14.9,13.2,6.5 \mathrm{~Hz}, \mathrm{C} \underline{\mathrm{H}}-17$ ), $2.10-2.19$ ( $\mathrm{m}, 1 \mathrm{H}, \mathrm{CH} \underline{\mathrm{H}}-9$ ), 2.30 (dt, $1 \mathrm{H}, J$ 19.1, $3.1 \mathrm{~Hz}, \underline{\mathrm{C}} \underline{\mathrm{H}}-8), 2.84$ (ddd, $1 \mathrm{H}, J$ 14.9, 13.2, $1.6 \mathrm{~Hz}, \mathrm{CH} \underline{\mathrm{H}}-17$ ), 3.07 (d, $1 \mathrm{H}, \mathrm{J}$ $11.4 \mathrm{~Hz}, \mathrm{C} \underline{\mathrm{H}}-5$ ), 3.72 (ddd, $1 \mathrm{H}, J 19.1,12.8,1.7 \mathrm{~Hz}, \mathrm{CH} \underline{\mathrm{H}}-8$ ), 3.98 (q, $2 \mathrm{H}, J 7.0 \mathrm{~Hz}, \mathrm{OCH}_{2}$ ), 4.50 (dd, $1 \mathrm{H}, J 11.4$, $0.9 \mathrm{~Hz}, \mathrm{CH} \underline{\mathrm{H}}-5), 4.69$ (dd, $1 \mathrm{H}, J$ 11.6, $1.1 \mathrm{~Hz}, \underline{\mathrm{C}} \underline{\mathrm{H}}-3), 4.79$ (d, $1 \mathrm{H}, J 11.6 \mathrm{~Hz}, \mathrm{CH} \underline{\mathrm{H}}-3), 6.80-6.88(\mathrm{~m}, 4 \mathrm{H}, \mathrm{ArH})$, 
7.10 - $7.15\left(\mathrm{~m}, 2 \mathrm{H}, \mathrm{C}_{6} \mathrm{H}_{5}\right), 7.37\left(\mathrm{tt}, 1 \mathrm{H}, \mathrm{J} 7.3,1.2 \mathrm{~Hz}, \mathrm{C}_{6} \mathrm{H}_{5}\right), 7.43-7.49\left(\mathrm{~m}, 2 \mathrm{H}, \mathrm{C}_{6} \mathrm{H}_{5}\right)$ ppm. ${ }^{13} \mathrm{C} \mathrm{NMR}(100 \mathrm{MHz}$, $\left.\mathrm{CDCl}_{3}, 23^{\circ} \mathrm{C}\right): \delta \mathrm{ppm}=14.91\left(\mathrm{CH}_{3}\right), 19.48,21.66,21.73,22.04,22.50,23.31,26.42,26.49\left(8 \times \mathrm{CH}_{2}\right), 34.25\left(\mathrm{CH}_{2}-\right.$ 8), $36.60\left(\mathrm{CH}_{2}-17\right), 51.70\left(\mathrm{CH}_{2}-5\right), 63.91\left(\mathrm{OCH}_{2}\right), 64.89$ (C-6), $68.57\left(\mathrm{CH}_{2}-3\right), 115.54,116.88(\operatorname{ArH}), 126.29,128.31$, $129.97\left(\mathrm{C}_{6} \mathrm{H}_{5}\right), 141.80,145.49\left(\mathrm{Ar}, \mathrm{C}_{6} \mathrm{H}_{5}\right), 153.27(\mathrm{Ar}), 202.97(\mathrm{C}=\mathrm{S}), 205.73(\mathrm{C}=\mathrm{O}) \mathrm{ppm}$. Anal. Calcd for $\mathrm{C}_{29} \mathrm{H}_{38} \mathrm{~N}_{2} \mathrm{O}_{2} \mathrm{~S}: \mathrm{C}, 72.76 ; \mathrm{H}, 8.00 ; \mathrm{N}, 5.85 ; \mathrm{S}, 6.70$. Found: $\mathrm{C}, 72.38 ; \mathrm{H}, 8.27 ; \mathrm{N}, 5.73 ; \mathrm{S}, 6.80 \%$.

\section{Procedure for the synthesis of 2,4-bis-(4-methylphenyl)-2,4-diazaspiro[5.5] undecan-7-one (5) in DMSO}

From $p$-tolylamine $(0.32 \mathrm{~g}, 3 \mathrm{mmol})$, cyclohexanone $0.1 \mathrm{~g}(1 \mathrm{mmol})$, and formaldehyde $(6 \mathrm{mmol}, 37 \%$ aqueous solution) according to the procedure described above for the three-component reaction (compounds $4 \mathbf{4 a}-\mathbf{I}$ ).

Procedure for the synthesis of 2,4-bis-(4-methylphenyl)-2,4-diazaspiro[5.5] undecan-7-one (5) in EtOH

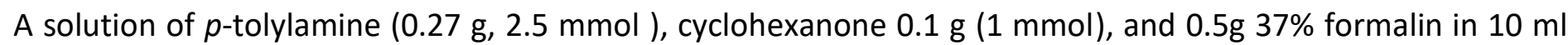
ethanol was treated under stirring with 5-10 drops of $L(+)$ - or DL-lactic acid. Stirring was continued $1 \mathrm{~h}$ at room temperature and the mixture was left standing until precipitation was complete (3-5h). The crystalline product was filtered off and recrystallized from ethanol. White crystals; yield $80 \%$ (DMSO), $42 \%$ (EtOH); $\mathrm{mp} 124-126^{\circ} \mathrm{C}$ (from EtOH); Lit. ${ }^{10} \mathrm{mp} 124-125^{\circ} \mathrm{C}$; Lit. ${ }^{11} \mathrm{mp} 124-126^{\circ} \mathrm{C}$. IR (ATR, $\mathrm{V}_{\max } \mathrm{cm}^{-1}$ ): 2932, 2860, 2817 (CH) 1703 (CO). MS (El, $70 \mathrm{ev}): m / z\left(\% I_{\max }\right)=348(41)[\mathrm{M}]^{+*}, 347$ (61), 119 (100), 118 (31), 91 (37).

Procedure for the synthesis of 1,3,5-tris-(4-bromophenyl)-1,3,5-triazinane (6) and $N, N^{\prime}$-bis-(4-nitrophenyl)-

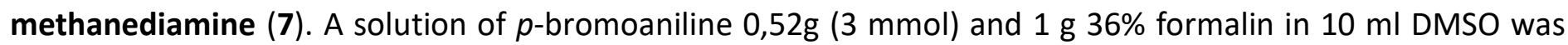
treated under stirring with $1 \mathrm{ml} \mathrm{L(+);} \mathrm{or} \mathrm{DL-lactic} \mathrm{acid.} \mathrm{Stirring} \mathrm{was} \mathrm{continued} 1 \mathrm{~h}$ at room temperature and, when spontaneous precipitation commenced, at $50-60^{\circ} \mathrm{C}$ for 2-3 h. Upon dilution with water some solid $\mathrm{NaHCO}_{3}$ was added to make precipitation complete. The crude product was dissolved in warm ethyl acetate and the solution was filtered in order to remove some solid contaminants. Evaporation of the solvent deposited crude 6 which was purified by recrystallization from ethanol.

The $N, N^{\prime}$-bis(4-nitrophenyl)methanediamine (7) was prepared similarly. However, the reaction mixture was left standing overnight prior to further processing. The crude air-dried product was purified by recrystallization from $\mathrm{MeNO}_{2} / \mathrm{EtOH}=2: 1$.

Compounds 5, 6, and 7 were characterised on the basis of melting points, NMR and comparison with authentic samples.

1,3,5-Tris-(4-bromophenyl)-1,3,5-triazinane (6). White crystals; yield: 80\%; mp $169-171{ }^{\circ} \mathrm{C}$ (from EtOH); Lit. ${ }^{24}$ $\mathrm{mp} 168$ - $170{ }^{\circ} \mathrm{C}$; Lit. ${ }^{25} \mathrm{mp}$ 170-172 ${ }^{\circ} \mathrm{C}$. IR (ATR, $\mathrm{V}_{\max } \mathrm{Cm}^{-1}$ ): 3070, 3035, 2860, 2737, 1586, 1567, $1489,1455$.

$\boldsymbol{N}, \boldsymbol{N}^{\prime}$-Bis-(4-nitrophenyl)methanediamine (7): Yellow fluffy crystals; yield 95\%; mp 237-239 ${ }^{\circ} \mathrm{C}$ decomp, (from $\left.\mathrm{MeNO}_{2} / \mathrm{EtOH}=2: 1\right) . \mathrm{Lit}^{26} 233^{\circ} \mathrm{C} . \mathrm{IR}\left(\mathrm{ATR}, \mathrm{V}_{\max } \mathrm{Cm}^{-1}\right): 3357(\mathrm{NH}), 1600,1530,1498,1466 \mathrm{~cm}^{-1}$.

\section{Acknowledgements}

We express our thanks to Professor Jerzy Lange of the Warsaw University of Technology for helpful discussions and assistance with the manuscript.

\section{Supplementary Material}

${ }^{1} \mathrm{H}$ and ${ }^{13} \mathrm{C}$ NMR spectra (pp. S2-S37) and elemental analyses (pp. S38-S45) are provided for compounds $\mathbf{2 - 4}$ in the Supplementary Data File. 


\section{References}

1. Ramón, D. J.; Yus, M. Angew. Chem., Int. Ed. 2005, 44, 1602.

https://doi.org/10.1002/anie.200460548

2. Chanda, A.; Fokin, V. V. Chem. Rev. 2009, 109, 725.

https://doi.org/10.1021/cr800448q

3. Zhou, L.; Bohle, D. S.; Jiang, H. F.; Li, C. J. Synlett 2009, 62, 75.

4. Pellissier, H., Asymmetric Domino Reactions, RSC Catalysis Series No. 10, The Royal Society of Chemistry 2013, Thomas Graham House, Science Park, Milton Road, Cambridge CB4 OWF, UK.

5. Kleinmann, E. F. in Comprehensive Organic Synthesis; Trost, B. M., Ed., Pergamon Press: New York, 1991, vol.2, chapter 4.1 .

6. Kobayashi, S.; Ishitani, H. Chem. Rev. 1999, 99,1069.

https://doi.org/10.1021/cr980414z

7. Córdova, A. Acc. Chem. Res. 2004, 37, 102.

https://doi.org/10.1021/ar030231l

8. Marques, M. M. B. Angew. Chem., Int. Ed. 2006, 45, 348.

https://doi.org/10.1002/anie.200502630

9. Mukhopadhyay, C.; Rana, S.; Butcher, R. J. Tetrahedron Lett. 2011, 52, 4153 and references therein. https://doi.org/10.1016/j.tetlet.2011.05.144

10. Wei H.-L.; Yan, Z.-Y.; Niu, Y.-N.; Li, G.-Q.; Liang Y.-M. J. Org. Chem. 2007, 72, 8600.

https://doi.org/10.1021/jo7016235

11. Dandia, A.; Jain, A.K.; Sharma, S. RSC Adv. 2013, 3, 2924.

https://doi.org/10.1039/c2ra22477a

12. Nayeem, A.; Saima, T.; Zeba, N. S. RSC Adv. 2015, 5, 50691.

https://doi.org/10.1039/C5RA08160B

13. Molvi, K. I.; Haque, N.; Bahlul Z. S. Awen, B.Z.S.; Zameeruddin, M. World J. Pharm. Pharm. Sci. 2014, 3, 536 and references therein.

14. Blunt, J.W.; Copp, B.R.; Keyzers, R.A.; Munro, M.H.G.; Prinsep, M.R. Nat. Prod. Rep. 2012, $29,144$. https://doi.org/10.1039/C2NP00090C

15. Daly, J.W.; Garaffo, H.M.; Spande, T.F.; Decker, M.W.; Sullivan, J.P.; Williams, M. Nat. Prod. Rep. 2000, 17, 131.

https://doi.org/10.1039/C2NP00090C

16. Jagodziński, T.S., Westerlich, S. Arkivoc 2013, (iii), 294.

http://dx.doi.org/10.3998/ark.5550190.p007.912

17. Jagodziński, T.S. Chem.Rev. 2003, 103, 197.

https://doi.org/10.1021/cr0200015

18. Sośnicki, J.G.; Struk, Ł. Synlett 2010, 8, 1209.

https://doi.org/10.1055/s-0029-1219792

19. Ried, W.; Käppler, W. Justus Liebigs Ann. Chem. 1964, 673, 132.

https://doi.org/10.1002/jlac.19646730119

20. Dandia, A.; Jain, A.K.; Sharma, S. Tetrahedron Letters 2012, 53, 5270.

https://doi.org/10.1016/i.tetlet.2012.07.079

21. Hünig, S.; Hoch, H. Chem. Ber. 1972, 105, 2197. 
https://doi.org/10.1002/cber.19721050713

22. Bossio, R.; Marcaccini, S.; Pepino, R.; Torroba, T. J. Chem. Soc. Perkin Trans. 1 1996, 229. https://doi.org/10.1039/p19960000229

23. Lamazouere, A.M.; Sotiropoulos, J. Tetrahedron, 1981, 37, 2451. https://doi.org/10.1016/S0040-4020(01)88901-8

24. Dandia, A., Jain, A.K., Sharma, S. Chemistry Letters 2014, 43, 521. https://doi.org/10.1246/cl.130839

25. Zhong, X.; Dou, G. Heterocycles, 2013, 87, 877. https://doi.org/10.3987/COM-13-12672

26. Zinner, H., Wigert, H. Chem. Ber. 1961, 94, 2209. https://doi.org/10.1002/cber.19610940840 